\title{
Whole-Food Plant-Based Lifestyle Program and Decreased Obesity: A 10-Year Follow-up
}

\author{
Boštjan Jakše ${ }^{1}$, Barbara Jakše ${ }^{2}$, Stanislav Pinter ${ }^{3}$, Jernej Pajek ${ }^{4}$ and Nataša Fidler Miss \\ 1 Biotechnical Faculty, University of Ljubljana, Jamnikarjeva 101, 1000 Ljubljana, Slovenia \\ 2 Barbara Jakše s.p., 1230 Domžale, Slovenia \\ 3 Faculty of Sport, University of Ljubljana, Gortanova 22, 1000 Ljubljana, Slovenia \\ 4 Department of Nephrology, University Medical Centre Ljubljana, Zaloška 2, 1525 Ljubljana, Slovenia \\ 5 Department of Gastroenterology, Hepatology and Nutrition, University Children's Hospital, University \\ Medical Centre Ljubljana, Bohoričeva 20, 1000 Ljubljana, Slovenia \\ * Correspondence: e-mail: natasa.fidler@kclj.si; Tel.: + 386- 1- 522- 3739
}

\begin{abstract}
Failure of various weight-loss programs and long-term maintenance of favorable body composition in all kinds of people is high, since the majority go back to old dietary patterns. Many studies have documented the efficacy of a plant-based diet (PBD) for body mass management, but there are opinions that maintaining a PBD is difficult. We aimed to evaluate the long-term success of a whole-food plant-based (WFPB) lifestyle program. We investigated the differences in the obesity indices and lifestyle of 151 adults (39.6 \pm SD 12.5 years), who were on our program for short $(0.5-<2$ years), medium ( $2-<5$ years), or long term (5-10 years). Body-composition changes were favourable for all three groups, both genders and all participants. There were no differences in relative bodycomposition changes (BMI, body fat percentage and muscle mass index (MMI)) between the three groups. All participants improved their BMI (baseline mean pre-obesity BMI range $\left(\mathrm{kg} / \mathrm{m}^{2}\right): 26.4 \pm 5.6$ to normal $23.9 \pm 3.8, \mathrm{p}<0.001)$, decreased body mass $(-7.1 \pm 8.3 \mathrm{~kg}, \mathrm{p}<0.001)$ and body fat percentage $(-6.4 \pm 5.6 \%$ points, $\mathrm{p}<0.001)$. Those with the highest BMI at baseline lost the most of: a) BMI units, $\mathrm{b})$ total body mass and c) body fat (a) $\left(\mathrm{kg} / \mathrm{m}^{2}\right)(-5.6 \pm \mathrm{SD} 2.9,-2.4 \pm 1.8$ and $\left.-0.9 \pm 1.5), \mathrm{b}\right)(\mathrm{kg})(-16.1 \pm \mathrm{SD}$ 8.8, $-7.1 \pm 5.4$ and $-2.5 \pm 4.5)$ and c) (\% points) $(-9.5 \pm$ SD 5.7, $-6.6 \pm 4.6$ and $-4.7 \pm 5.3)$ for participants who had baseline BMI in obese, overweight and normal range, respectively; pbaseline vs. current $<0.001$ for all). $85.6 \%$ (101 out of 118 ) of parents of underage children ( $<18$ years), introduced WFPB lifestyle to their children. WFPB lifestyle program provides long-term lifestyle changes for reversal of obesity and is effective transferred to the next generation.
\end{abstract}

Keywords: nutrition; plant-based diet; vegan diet; lifestyle; obesity; body composition; weight-loss.

\section{Introduction}

In recent years, adopting a strict plant-based (vegan) diet (PBD) that is appropriately planned and supervised has become increasingly popular. The health effects of a well-designed strict PBD have been recognized in several dietetic communities [1-7]. Furthermore, numerous professional organizations on food and nutrition also state that an appropriately planned vegetarian diets (often interchangeably named as PBDs), including a vegan diet (strict PBD), are appropriate for all stages of 
the life, including pregnancy, lactation, infancy, childhood, adolescence, and older adulthood $[1,3,4]$, as well as for athletes $[1,2]$.

From prospective studies, it is known that plant-based dieters have healthier lifestyle habits than nonvegetarians [8-10], so it was assumed that the health effects of PBDs were strongly associated with the accompanying healthier lifestyle [11-20]. Many previous studies on long-term strict plantbased dieters that were exploring the impact of the diet and lifestyle factors on health frequently reported healthier lifestyle behaviours [10,21-23]. A strict PBD (for the purpose of this paper, strict PBD will serve as a substitution for vegan diet) was also found to be associated with better mental health, where vegans reported less stress and anxiety, and better mood status compared to omnivores [9].

Adopting a strict PBD is demanding, and many individuals might face problems and obstacles (e.g., facing social pressure and expected norms, unadjusted lifestyle for the necessary changes, lack of cooking skills, and having limited eating options when outside home or at work) in achieving and maintaining this goal. Long-term compliance, adherence to prescribed diet plans, and preservation of body mass reduction is a significant concern with weight-loss interventions, which is often one of the major motives to adopt a strict PBD. In brief, the rate of failure of various weightreducing interventions and the probability of attaining the target body mass and long-term maintaining body mass loss in all obese people is high, and may go up to $99.8 \%$ [24,25]. In metaanalysis of 29 long-term weight-loss studies on individuals in U.S. completing a structured body mass-loss program, over half of the lost body mass was regained by two years, and by five years approximately $80 \%$ of the lost body mass was regained [26]. Although adherence to a strict PBD appears to be similar to most other therapeutic dietary approaches [27], the transition to a strict PBD may be more successful with inclusion of participants in a lifestyle-optimizing program providing a rationale for a diet change through lectures, prescribed daily meal plans and feedback, individual and group counselling, and social support, including grocery-shopping tours, cooking workshops, frequent body-composition measurements, and motivational sessions [28-32].

To become long-term motivated healthy plant-based dieters, individuals need to change several important lifestyle behaviours. A healthy and active lifestyle is associated with health and quality of life [33], while an unhealthy lifestyle is a recognized major risk factor for the development of various chronic diseases and premature mortality [34,35]. Physical activity (PA) [36], sleep hygiene [37], and stress status [38] are well-established modifiers that play a role in health and disease prevention. This and many others healthy-lifestyle factors (e.g., moving naturally, having purpose, belonging to a community, being in social circles that support healthy behaviours, investing in family happiness and care) were presented as keys for longevity in studies of centenarians across diverse geographical locations ("Blue Zone" areas) [39]. A fundamental healthy lifestyle includes having healthy nutrition, maintaining a proper body mass, avoiding smoking, limiting alcohol drinking, and being physically active, which might account for up to $50 \%-60 \%$ reduced risk of all-cause mortality in men and women [40]. There is overwhelming evidence supporting the health benefits of PA or exercise. PA or exercise has an important role in body mass management, particularly when combined with dietary changes $[41,42]$. However, the PA level alone also has independent positive impact on other aspects of health [43].

Ten years ago, we developed a whole-food plant-based (WFPB) lifestyle program that included three proven approaches: (1) the nutritional part (supplemented WFPB diet (Supp WFPB 
diet)), (2) the PA component, and (3) a support system that included a social-media component (described under Materials and Methods). With such an extensive lifestyle program based on WFPB diet, it may be easier to maintain healthier long-term normal body mass, body composition, and health. Previously we reported the effects of our WFPB lifestyle program on body-composition indices during 10-weeks [44], and cardiovascular risk factors during 10-36 weeks [28].

In the present cross-sectional study we evaluated lifestyle and body composition indices (body mass index (BMI) and body composition) of participants from Slovenia who were on our WFPB lifestyle program [28] for 0.5-10 years. Our hypothesis was that there is significant difference in the body-composition indices between participants who were in our program for the short $(0.5-<2$ years) vs. medium term ( $2-<5$ years), but not between those in it for the medium vs. long term (5-10 years) $\left(\mathrm{H}_{0}\right)$. To test this, we divided participants into three groups, according to their stay in our program: short, medium, and long term. Our aim was also to evaluate changes in obesity markers (BMI units, total body mass and body fat percentage) for participants from the time of entering our program as practising the Western-type diet (baseline) to the present (0.5-10 years on our WFPB lifestyle program).

\section{Materials and Methods}

\subsection{Study Design and Eligibility}

We included free-living, heterogeneous participants from different parts of Slovenia who had been in our WFPB lifestyle program, described previously [28] for 0.5-10 years, but also included some of their baseline anthropometric measures (see Materials and Methods). In short, our extensive lifestyle program included the nutritional part, the PA component, and a support system. Participants were not remunerated financially to participate in the study. The study was conducted in accordance with the Declaration of Helsinki, and the protocol was approved by the Slovenian Medical Ethics Committee (approval document 0120-380/2019/17) and the Slovenian Ethical Committee in the field of sports (approval document No. 05:2019). This trial was registered 6.6.2019 at https://clinicaltrials.gov with number NCT03976479.

\subsection{Subjects}

We invited 2555 adults, aged 18-80 years, through our closed social-media support groups and by personal contact with PBD health coaches. All participants were in the same support system, but individuals could choose where they would be on the PBD continuum, from plant-rich diet to a WFPB diet. Participants were also included if they had some dietary restrictions (e.g., gluten, tomatoes, peanuts, and citrus). Non-inclusion criteria were current pregnancy or lactation, current competitive or top-level athletes, major musculoskeletal restrictions, active malignant diseases (e.g., cancer, CVD, type 2 diabetes, autoimmune and neurodegenerative diseases), and the current use of drugs for measured blood markers (plasma lipids, blood pressure). Exclusion criteria were: $\geq 3 \%$ of energy intake from animal protein, incomplete blood assay and unanswered questionnaires. The majority of the invited adults were females, and 370 individuals met the inclusion criteria after 2phase interviews. In total, 204 declined to participate out of reasons like being stressed to participate in the study, vacation (summer time) period, demanding methods (evaluation of dietary intake by 3 day weighted dietary record (3-DR), discomfort against blood collection, or/and a challenging personal situation. We included everyone eligible by the inclusion criteria who responded to our invitation to participate in the study. 166 individuals signed the informed-consent form, out of which four discontinued to participate, we excluded six participants as they were still eating a 
significant amount of animal-based products on a regular basis based on 3-DR evaluation, two had an incomplete blood assay, two had unanswered questionnaires, while one did not want to complete them.

The study lasted from June until August 2019, but the clinical part of this study was finished within 14 days. In the final analysis, we included 151 adult participants (91\% of initially included; Figure 1). For the inclusion criteria, we did not set limits concerning current BMI since we had known their baseline body mass (BM) and body-fat percentage (BF \%) or smoking status. Past measurements were measured by three investigators and their teams of health coaches, and archived according to the General Data Protection Regulation (GDPR). Based on the duration of the WFPB lifestyle program, we divided participants into 3 groups: short-term $(0.5-<2$ years), medium-term $(2-<5$ years), and longterm (5-10 years).

\subsection{WFPB lifestyle program}

\subsubsection{Nutritional part}

The nutritional part included a 60 -min lecture/week for 10 weeks covering the rationale and with guidance on WFPB diet as well as healthy active lifestyle, group cooking workshops, recipes, and individual counselling with whole-food shopping guidance and individual meal plans, all with an experienced health coach who was leading by example. The dietary program consisted of $\geq 90 \%$ WFPB diet, while $0-10 \%$ of the energy intake was supplemented with nutrient-enriched plant-based meal replacement (MR; 35-37 g of plant protein (soy or pea)/100 g; 1-2 portions/day; 10-15 g of plant protein/portion) and dietary supplements (e.g., vitamin $\mathrm{B}_{12}$ for all, vitamin $\mathrm{D}_{3}$ (October-May), optionally n-3 long-chain polyunsaturated fatty acids (eicosapentaenoic acid (C20:5n-3, EPA) and docosahexaeneoic acid (C22:6n-3, DHA)) based on individual dietary needs, cooking skills, and lifestyle challenges. The WFPB diet in our dietary program was defined by Campbell and Campbell (2005). It allows ad libitum intake of whole grains, fruits, vegetables, and legumes, moderate intake of nuts, seeds, avocados, and soy (e.g., tofu) or wheat products with no added fat. It includes little or no added refined fat (e.g., olive oil, coconut, and palm oil). It excludes all animal products (like all vegan diets) and is based predominately on whole or minimally processed plant foods. Ultra-processed foods, as defined by the NOVA classification system [45], highly refined carbohydrates (e.g., white rice, white flour), foods containing added sugars (e.g., table sugar, high-fructose corn syrup), and sweeteners were omitted [46]. To ensure nutrient adequacy without adding excess energy [47], we included up to $10 \%$ of energy intake from MR to WFPB diet (Supp WFPB diet). We chose MR that was safe, simple to prepare, effective, convenient [48-52], and with the purpose of the better compliance [53]. Supp WFPB diet was individually optimized by an experienced health coach to meet nutritional needs, cultural preferences, and lifestyle. Despite the fact that body mass management was a core part of our program, there was no need for calorie counting, and the recommended dietary program was consumed ad libitum to full satiety at each meal [28]. During the introduction phase of our WFPB lifestyle program (the first 10 weeks), a small portion of foods of animal origin (i.e., meat, milk, eggs) was allowed once/week to relieve social pressures on participants from their circle of influence (i.e., family, friends, and co-workers). After the introduction phase, the intake of foods of animal origin was discouraged. It was only tolerated occasionally as a flavour commodity in meals with family/friends, if they were in unusual and unprepared situations when eating out of home, or still to relive social pressures from their circle of influence, but was limited to $<30 \mathrm{~g}$ animal-based foods/week.

\subsubsection{Physical activity component}


The PA component included habitual (part of participants' daily life), organized (guided or prescribed exercise), and not-organized (part of a healthy and active lifestyle) PA. During the introduction phase, the participants were encouraged to engage in at least two 45 min guided moderate-intensity exercise sessions/week to accompany their new dietary style with additional PA as a paradigm for a healthier lifestyle. The guided 45 min moderate-intensity group exercises in several cities in Slovenia were offered free of charge. After the introduction phase, the participants became more independent and performed the prescribed resistance-exercise activities by themselves. Participants were also encouraged to perform at least 30 minutes per day of low- to moderateintensity aerobic activity (brisk walking or biking) and a longer low- to moderate-intensity activity during the weekend ( $>45$ minutes, preferably 1-2 hours in 1 or 2 sessions), like brisk walking or hiking, both as part of a healthy active lifestyle.

\subsubsection{Support system}

The support system consisted of regular follow-ups and various support-system services, for example, frequent body-composition measurements, food dairy, grocery tour, cooking workshops, assistance in the introduction of regular and appropriate PA, individual and group support on various challenges during behavioural changes, and social-media support groups including four parts: 1) cooking recipes, 2) professional summaries of health and nutrition topics written in lay language, 3) posted organized group workouts and results/testimonials, and 4) discussion board. The discussion board provided answers to their dilemmas on controversies and common nutritional myths, particularly about WFPB diet and other weight-loss diets, as well as active social interactions. The goal of the social-media support group was to motivate participants to comply with the prescribed dietary regiment, to receive accurate information, to share new experiences and cooking skills with each other, and to help face daily challenges, all with the primary aim of improving their well-being and long-term health.

\subsection{Outcomes}

\subsubsection{Sociodemographic and Economic Status, and Motives for PBD}

For sociodemographic and economic status, we adopted the questionnaire for adults by the National Institute of Public Health [54] from the project called "EU Menu Slovenia", the European Food Safety Authority (EFSA) project EU Menu (see Table 1). We modified the questionnaire to include additional questions for those interested in WFPB diet, such as number of underage children ( $<18$ years) of the studied parents that are on WFPB lifestyle program, and number of children born by mothers adopting WFPB lifestyle program and their birth BM. For evaluating the motives for following strict PBD, we asked participants to rank 8 different motives (1 was the least important and 8 was the most important), namely, (1) health, (2) BM management/appearance, (3) environmental concerns, (4) religious reasons, (5) affordable dieting, (6) convenient dieting, (7) animal ethics, and (8) satiety/no hunger. For each motive, we calculated the average score and share from the whole sample.

\subsubsection{Everyday sitting, transport time, and $P A$}

To assess PA, inactivity, and time spent using passive transport we used the last 7 days with the self-administered International Long Physical Activity Questionnaire (L-IPAQ; [55] that was translated into the Slovenian language and reviewed by an experienced $\mathrm{PhD}$ Sport physiologist in this 
field. All study participants were instructed to maintain their pre-existing PA rhythm. Components of L-IPAQ that we emphasized in our study on PA status as outcome were (1) minutes of traveling in a motorized transport (e.g., car, bus, train), (2) time spent sitting during weekdays, (3) times spent sitting during the weekend, (4) average daily walking minutes, (5) average daily moderate PA minutes, (6) average daily vigorous PA minutes, and (7) total L-IPAQ score. The fourth to sixth outcomes were related to transportation, housework/gardening, recreation, sport, and leisure-time PA. The volume of activity was computed by weighting each type of activity by its energy requirements defined in METs. METs are multiples of the resting metabolic rates to yield a score in MET-minutes. In the calculation, the L-IPAQ scoring protocol assigns the following MET values: walking was considered to be 3.3 METs (3.3 multiple minutes of walking multiple times/week), moderate-intensity PA was considered to be 4 METs, and vigorous-intensity PA was defined as 8 METs. Total METs minutes of PA per week are achieved with a sum of MET minutes achieved in each category (walking, moderate activity, and vigorous activity [56]). One MET is defined as a resting metabolic rate obtained during quiet sitting and is equivalent to the caloric consumption of 1 $\mathrm{kcal} / \mathrm{kg} /$ hour [57]. High PA level is defined as vigorous-intensity PA on at least 3 days per week, achieving a minimum total PA of at least 1.500 METs minutes per week or 7 days on any combination of walking, moderate-intensity PA, or vigorous-intensity PA achieving a minimum total PA at least 3.000 MET minutes per week. Moderate PA level is classified as 5 or more days of any combination of the 3 PA categories, achieving a minimum total PA of 600 MET minutes per week [58]. Less than $0.5 \%$ of all data was rechecked for accuracy of entry as a result of potential input errors or missed data. We added a question to the L-IPAQ about weekly frequency of at least 30 minutes of organized resistance/weight-lifting workouts to further distinguish the PA lifestyle pattern according to general recommendations for PA.

\subsubsection{Sleep quality and patterns}

To assess sleep quality, patterns, and disturbance, and daytime dysfunction during the last month, we used 19 self-rated questions of the Pittsburgh Sleep Quality Index (PSQI) questionnaire [59]. We found 10 components of PSQI overall to be important for our study, namely, (1) time of going to sleep, (2) time of waking up, (3) subjective sleep quality, (4) sleep efficiency, (5) sleep latency, (6) sleep duration, (7) sleep disturbance, (8) use of sleep medications, (9) daytime dysfunction, and (10) global sleep-quality score (sum of 7 PSQI components scores from scoring rules). The total score range was from 0 to 21, where a higher score indicates worse sleep quality, and 5 or greater is indicative as a cut-off for poor sleep quality [59].

\subsubsection{Stress status}

To measure stress status during the last month (recent version), we used a 30-question Perceived Stress Questionnaire (PSQ) [60]. PSQ is a valid instrument for recording subjectively perceived stress in the context of a transactional view of stress [61] and stress-related diseases [62]. The PSQ emphasizes cognitive level of experience more than emotional states or specific life events. The items can be answered with a 4-point Likert-type rating scale $(1=$ almost never, $2=$ sometimes, $3=$ often, $4=$ usually). Factor analysis revealed 7 PSQ factors defining perceived stress: harassment, overload, irritability, lack of joy, fatigue, worries, and tension. It contains both positively and negatively formulated items in order to reduce acquiescent bias. After several positive items $(1,7,10$, $13,17,21,25,29)$, scores were calculated in reverse and then, summing all item scores, resulted in a total raw score range of 30 to 120. A PSQ index varying from 0 (lowest level of perceived stress) to 1 
(highest level of perceived stress), was derived from the total raw score using the formula: PSQ Index $=$ total raw score $-30 / 90[60,63]$. Cut-off values for levels of stress established on this basis were: $<0.34$ (low stress), 0.34-0.46 (moderate stress), and >0.46 (high stress) [64]. We translated PSQ into Slovenian language with additional help from an experienced psychologist in the cognitive stress filed.

\subsubsection{Anthropometric and Body Composition Measures}

All participants were measured without shoes, socks, outer clothing, mobile device and/or keys in pockets. Baseline data (at the entrance into our program) were measured by three investigators and their teams of health coaches, with same bio-electrical impedance analysis technology (Tanita, Japan) and archived according by the General Data Protection Regulation (GDPR). In the current study, all measurements were made by the same researcher with the assistance of another. Heights (cm) were measured by the body height gauge (MPE 250K100HM), Body composition was assessed by an 8-electrode medically approved and calibrated bio-electrical impedance body-composition monitor (Tanita 780 S MA, Tanita Corporation, Tokyo, Japan) which provides an accurate tool to measure total body fat percentage and fat free mass in healthy young males and females, regardless of their level of habitual PA [65]. Body mass index (BMI, $\left.\mathrm{kg} / \mathrm{m}^{2}\right)$ and muscle-mass index $\left(\mathrm{MMI}, \mathrm{kg} / \mathrm{m}^{2}\right)$ were calculated from body height and BM, and body height and muscle mass that were measured by body-height gauge and body-composition monitor. Body-composition indices included BM, BMI, body fat mass percentage $(\mathrm{BF} \%)$ relative to total $\mathrm{BM}$, visceral fat rating (in arbitrary units associated with the abdominal visceral fat cross-sectional area, where each unit equals $10 \mathrm{~cm}^{2}$ of visceral fat), muscle mass, MMI and total body water. Before the bio-impedance test, participants were asked not to eat or drink for at least 1 hour, exercise for at least $24 \mathrm{~h}$, and urinate for at least 30 minutes.

\subsubsection{Dietary intake and cardiovascular risk factors}

We have also evaluated dietary and supplements intakes by 3-DR as well as several cardiovascular risk factors, which we are going to present in a separate manuscript.

\subsection{Statistical Analysis}

Statistical analysis was done with R 3.5.2 with the dpylr [66], ggplot2 [67] and arsenal [68] packages. For numerical variables, we used ANOVA for the differences between different groups and the Tukey post hoc test, where differences were statistically significant. Where the subsample was small, we referred to the Kruskal Wallis test. When we analysed dependent samples, we used t-test for dependent sample.

Similar for the categorical variables, we used the chi-square test, and Fisher's exact test where the subsample was small. The threshold for statistical significance was 0.05 .

\section{Results:}

\subsection{Participant Characteristics}

The whole sample $(n=151)$ included 109 females $(72 \%)$ and 42 males $(28 \%)$ from six regions of Slovenia. The age (mean \pm SD) and current BMI (mean (min-max)) of the participants was $39.6 \pm 12.5$ years and $23.9(17.7-41.4) \mathrm{kg} / \mathrm{m}^{2}$. Group 1 included 51 participants (35 females (69\%) and 16 males $(31 \%))$, Group 2 included 56 participants (43 females (77\%) and 13 males (23\%)), and Group 3 included 44 participants (31 females $(70 \%)$ and 13 males $(30 \%)$ ). Most participants lived in a marriage or partnership status, outside the capital $(62.3 \%)$, lived in a suburban $(31.8 \%)$ or rural $(41.1 \%)$ 
environment, were employed or self-employed (79.4\%), and rated themselves as having at least mildly above-average economic status (94.7\%). The majority of participants were college educated (62.2\%). Participants in our study had 196 children, 118 were underage ( $<18$ years; 60\% of all children), while 22 were adult ( $>18$ years; $40 \%$ of all children). $85.6 \%$ of underage children $(n=101$ of 118$)$ and $28.2 \%$ of adult children $(n=22$ of 78$)$ were on WFPB diet. $8.2 \%$ children $(n=16)$ were born to parents while being on our WFPB diet lifestyle program. During pregnancy, 7 women gained 7-10 kilograms (mean BM of their infants: 3683 grams), 8 women gained 10.1-12 kilograms (mean BM of their infants: 3477 grams) and one women gained 12.1-15 (mean BM of her infant: 3270 grams). Current main sociodemographic and economic characteristics are shown in Table 1 and more detailed in supplementary table Table $1 S$.

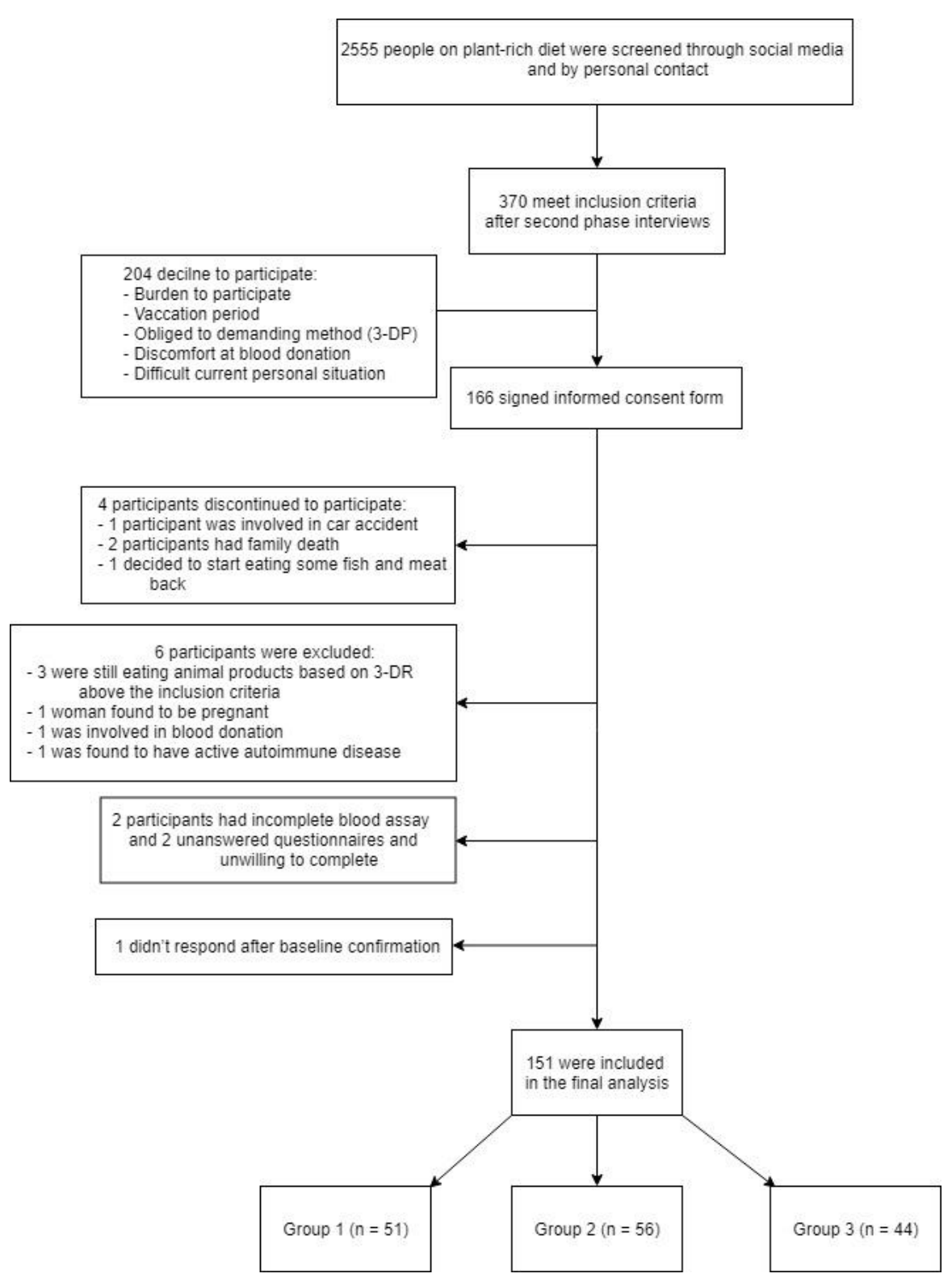

Figure 1. Enrolment of participants and study completion. 
Table 1. Current demographic and other characteristics of all participants and according to their time in WFPB lifestyle program: short $(0.5-<2$ years), medium ( $2-<5$ years $)$ and long-term (5-10 years) groups (1, 2 and 3).

\begin{tabular}{|c|c|c|c|c|c|c|c|}
\hline \multirow{2}{*}{$\begin{array}{l}\text { Parameter } \\
\text { Age (years) }\end{array}$} & \multirow{2}{*}{$\begin{array}{c}\text { Whole sample }(\mathbf{n}=151) \\
39.6 \pm 12.5\end{array}$} & \multicolumn{2}{|c|}{ Group $1(n=51)$} & \multicolumn{2}{|c|}{ Group $2(n=56)$} & \multicolumn{2}{|c|}{ Group $3(n=44)$} \\
\hline & & $36.9 \pm 11.5$ & $37.3 \pm 17.6$ & $42.6 \pm 10.9$ & $36.1 \pm 10.3$ & $42.4 \pm 13.5$ & $35.8 \pm 11.0$ \\
\hline \multirow[t]{2}{*}{ Gender $(n, \%)$} & Both genders & Female & Male & Female & Male & Female & Male \\
\hline & $(100 \%)$ & $35(69 \%)$ & $16(31 \%)$ & $43(77 \%)$ & $13(23 \%)$ & $31(70 \%)$ & $13(30 \%)$ \\
\hline Time on (WFPB) lifestyle program (years) & $4.1(0.8-10)$ & $1.3(0.9-1.9)$ & $1.2(0.8-1.8)$ & $3.9(2.5-4.9)$ & $4.0(2.5-4.9)$ & $6.9(5.5-10.0)$ & $7.2(5.5-10.0)$ \\
\hline Height $(\mathrm{cm})$ & $169.0 \pm 8.6$ & $167.7 \pm 7.6$ & $178.5 \pm 5.3$ & $164.2 \pm 5.0$ & $178.8 \pm 4.0$ & $163.5 \pm 4.8$ & $179.8 \pm 6.9$ \\
\hline \multicolumn{8}{|l|}{ Smoking (n (\%)) } \\
\hline Never & $117(77.5 \%)$ & & & & & & \\
\hline Former & $7(4.6 \%)$ & & & & & & \\
\hline Current & $26(17.2 \%)$ & & & & & & \\
\hline N/A (did not disclose) & $1(0.7 \%)$ & & & & & & \\
\hline Alcohol (n (\%)) & $3(2 \%)$ & & & & & & \\
\hline Alcohol (g/d) & 0.4 & & & & & & \\
\hline \multicolumn{8}{|l|}{ Participants' children } \\
\hline Children of participants (n) & 196 & & & & & & \\
\hline Underage children ( $<18$ years) $(\mathrm{n},(\%$ of all children) & $118(60 \%)$ & & & & & & \\
\hline Adult children (>18 years) ( $\mathrm{n}$ (\% of all children) & $78(40 \%)$ & & & & & & \\
\hline Underage children ( $<18$ years) on WFPB diet ( $n$, (\% of underage)) & $101(85.6 \%)$ & & & & & & \\
\hline Adult children (>18 years) on WFPB diet $(\mathrm{n},(\%$ of adult children $))$ & $22(28.2 \%)$ & & & & & & \\
\hline Children born to parents while on WFPB diet lifestyle ( $n$, $(\%$ of all children)) & $16(8.2 \%)$ & & & & & & \\
\hline Pregnancy BM gainp & $\mathrm{N}$ (Infant's birth BM) & & & & & & \\
\hline $7-10 \mathrm{~kg}$ & $7(3683 \mathrm{~g})$ & & & & & & \\
\hline $10.1-12 \mathrm{~kg}$ & $8(3477 \mathrm{~g})$ & & & & & & \\
\hline $12.1-15 \mathrm{~kg}$ & $1(3270 \mathrm{~g})$ & & & & & & \\
\hline
\end{tabular}

Data are means \pm standard deviation (SD) for normally distributed variables and medians (minimum-maximum) for non-normally distributed ones. Whole-food plant-based

(WFPB). ${ }^{+}$Maximal reported mass that a participant reached at any time during their life. PData for their time during pregnancy, not currently. 


\subsection{Lifestyle Factors}

The whole sample presented relatively low weekly $(5.0 \pm 2.9$ hours $)$ and weekend $(4.0 \pm 2.3$ hours) prolonged daily siting, and high PA (1902 METS of vigorous-intensity PA and 5542 METS of total PA) that includes regular resistance workout with their own BM or by a prescribed fitness program. Assessment of sleep quality showed that the whole sample had good sleeping quality. Participants went to sleep very differently (most of them before 22:00 (33.8\%) or after midnight $(37.7 \%))$, and woke up before $6: 30(83.4 \%)$, evaluated sleep quality as very and fairly good (143, $94.7 \%)$, fell asleep within 15 minutes (121, 80.1\%), they slept over 6 hours per night $(114,75.5 \%)$, were very sleep-efficient $(146,96.7 \%)$, had less than once per week or no sleep disturbance $(140,92.7 \%)$, only 3 participants used sleep medications during the past month, 93 participants (61.6\%) and 50 participants (33.1\%) had 1-2 and 3-4 occasions of daytime dysfunctions, and the whole sample had a good overall total score of sleep quality $(2.7 \pm 3.9)$. The studied sample also showed a favourable positive subscale score (i.e., "how often have you felt that things were going your way?") and total score $(P S Q=0.3 \pm 0.1)$ that indicated lower stress level. All measures were nonsignificant different in the group comparison (Table 2). 
Table 2: Current lifestyle factors of all participants and according to their time in WFPB lifestyle program: short (0.5-<2 years), medium (2-<5 years) and long-term (5-10 years) groups (1, 2 and 3).

\begin{tabular}{|c|c|c|c|c|c|}
\hline Parameter & Whole sample & Group $1(n=51)$ & Group $2(n=46)$ & Group $3(n=44)$ & $p$-value \\
\hline \multicolumn{6}{|c|}{ International Physical Activity (PA) - Long (IPAQ score) } \\
\hline Weekly sitting (h/d) & $5 \pm 3$ & $4 \pm 3$ & $5 \pm 3$ & $5 \pm 2$ & NS \\
\hline Walking PA (METS min/w) & $1488 \pm 1811$ & $1300 \pm 1261$ & $1544 \pm 2234$ & $1634 \pm 1773$ & NS \\
\hline Moderate-intensity PA (METS min/w) & $2171 \pm 2365$ & $1906 \pm 1712$ & $1972 \pm 2283$ & $2731 \pm 2997$ & NS \\
\hline Vigorous PA (METS min/w) & $1902 \pm 2819$ & $2440 \pm 4063$ & $1447 \pm 1839$ & $1857 \pm 1892$ & NS \\
\hline \multicolumn{6}{|c|}{ Sleep Quality (PSQI score) } \\
\hline \multicolumn{5}{|c|}{ Sleep time $(\mathrm{n}(\%))$} & NS \\
\hline \multicolumn{6}{|l|}{ Time } \\
\hline 21:01-22:00 & $51(33.8 \%)$ & $17(33.3 \%)$ & $14(25.0 \%)$ & $20(45.5 \%)$ & \\
\hline 22:01-23:00 & $25(16.6 \%)$ & $6(11.8 \%)$ & $12(21.4 \%)$ & $7(15.9 \%)$ & \\
\hline 23:01-00:00 & $18(11.9 \%)$ & $5(9.8 \%)$ & $6(10.7 \%)$ & $7 / 15.9 \%)$ & \\
\hline $5: 31-6: 30$ & $68(45 \%)$ & $24(47.1 \%)$ & $26(46.4 \%)$ & $18(40.9 \%)$ & \\
\hline 6:21-6:59 & $3(2 \%)$ & $1(2 \%)$ & 0 & $2(4.5 \%)$ & \\
\hline From 7:00 & $22(14.6 \%)$ & $7(13.7 \%)$ & $6(10.7 \%)$ & $9(20.5 \%)$ & \\
\hline \multicolumn{5}{|c|}{ Subjective sleep quality } & NS \\
\hline Very and fairly good & $143(94.7 \%)$ & $48(94.1 \%)$ & $53(94.6 \%)$ & $44(95.5 \%)$ & \\
\hline Fairly and very bad & $8(5.3 \%)$ & $3(5.9 \%)$ & $3(5.4 \%)$ & $2(4.5 \%)$ & \\
\hline \multicolumn{5}{|c|}{ Sleep latency $(\mathrm{min})$} & NS \\
\hline$\leq 15 \mathrm{~min}$ & $121(80.1 \%)$ & $38(74.5 \%)$ & $47(83.9 \%)$ & $36(81.8 \%)$ & \\
\hline $16-30 \mathrm{~min}$ & $26(17.2 \%)$ & $11(21.6 \%)$ & $712.5 \%)$ & $8(18.2 \%)$ & \\
\hline
\end{tabular}




\begin{tabular}{|c|c|c|c|c|c|}
\hline Parameter & Whole sample & Group $1(n=51)$ & Group $2(n=46)$ & Group $3(n=44)$ & p - value \\
\hline $31-60 \mathrm{~min}$ & $4(2.6 \%)$ & $2(3.9 \%)$ & $2(3.6 \%)$ & 0 & \\
\hline \multicolumn{5}{|c|}{ Sleep duration $(\mathrm{h})$} & NS \\
\hline More than 7 hours & $44(29.1 \%)$ & $18(35.3 \%)$ & $12(21.4 \%)$ & $14(31.8 \%)$ & \\
\hline 6-7 hours & $70(46.4 \%)$ & $21(41.2 \%)$ & $28(50.0 \%)$ & $21(47.7 \%)$ & \\
\hline Fewer than 6 hours & $37(24.5 \%)$ & $12(23.5 \%)$ & $16(28.6 \%)$ & $9(20.5 \%)$ & \\
\hline \multicolumn{5}{|c|}{ Sleep efficiency (score) } & NS \\
\hline $65 \%-74 \%$ & $2(1.3 \%)$ & 0 & $1(1.8 \%)$ & $1(2.3 \%)$ & \\
\hline $75 \%-84 \%$ & $3(2 \%)$ & 0 & 0 & $3(6.8 \%)$ & \\
\hline$>85 \%$ & $146(96.7 \%)$ & $51(100.0 \%)$ & $55(98.2 \%)$ & $40(90.9 \%)$ & \\
\hline \multicolumn{5}{|c|}{ Sleep disturbance } & NS \\
\hline Not during the past month & $16(10.6 \%)$ & $5(9.8 \%)$ & $9(16.1 \%)$ & $2(4.5 \%)$ & \\
\hline Less than once a week & $124(82.1 \%)$ & $41(80.4 \%)$ & $44(78.6 \%)$ & $39(88.6 \%)$ & \\
\hline Once or twice a week & $10(6.6 \%)$ & $5(9.8 \%)$ & $3(5.4 \%)$ & $2(4.5 \%)$ & \\
\hline Three or more times a week & $1(0.7 \%)$ & 0 & 0 & $1(2.3 \%)$ & \\
\hline \multicolumn{5}{|c|}{ Sleep mediation use } & NS \\
\hline Not during the past month & $148(98 \%)$ & $50(98.0 \%)$ & $55(98.2 \%)$ & $43(97.7 \%)$ & \\
\hline Less than once a week & $1(0.7 \%)$ & $1(2 \%)$ & 0 & 0 & \\
\hline Three or more times a week & $2(1.3 \%)$ & 0 & $1(1.8 \%)$ & $1(2.3 \%)$ & \\
\hline \multicolumn{5}{|c|}{ Daytime dysfunction (n/w) } & NS \\
\hline 1-2 days & $93(61.6 \%)$ & $34(66.7 \%)$ & $33(58.9 \%)$ & $26(59.1 \%)$ & \\
\hline 3-4 days & $50(33.1 \%)$ & $12(23.5 \%)$ & $21(37.5 \%)$ & $17(38.6 \%)$ & \\
\hline 5-6 days & $5(5.3 \%)$ & $4(7.8 \%)$ & $1(1.8 \%)$ & 0 & \\
\hline Every day & $3(2 \%)$ & $1(2 \%)$ & $1(1.8 \%)$ & $1(2.3 \%)$ & \\
\hline Global sleep quality & $2.7 \pm 1.8$ & $2.8 \pm 1.7$ & $2.5 \pm 1.8$ & $2.7 \pm 2.1$ & \\
\hline \multicolumn{6}{|c|}{ Perceived Stress Questionnaire (PSQ score) } \\
\hline Positive items score & $17.3 \pm 3.9$ & $17.0 \pm 3.9$ & $17.9 \pm 4.2$ & $16.8 \pm 3.8$ & NS \\
\hline Total score & $0.3 \pm 0.1$ & $0.3 \pm 0.1$ & $0.3 \pm 0.1$ & $0.3 \pm 0.1$ & NS \\
\hline
\end{tabular}

Data are mean \pm standard deviation (SD) for normally distributed variables. 


\subsection{Motives for Adopting PBD}

The health motive for adopting WFPB dietary pattern was the most prevalent choice, since 141 participants (93.4\%) of the whole sample ranked this motive as 8 on scale of 1-8 (from least to most important). Mass management/appearance motive was chosen by 95 participants (62.9\%) as 7 . The third most important motive for adopting WFPB dietary pattern was satiety/no hunger (score was 4.8 \pm 1.7 ), which was followed by convenient dieting (score was $4.3 \pm 1.3$ ) and environmental concerns (score was $4.1 \pm 1.8$ ). Motives for adopting WFPB dietary pattern are shown in Figure 2.

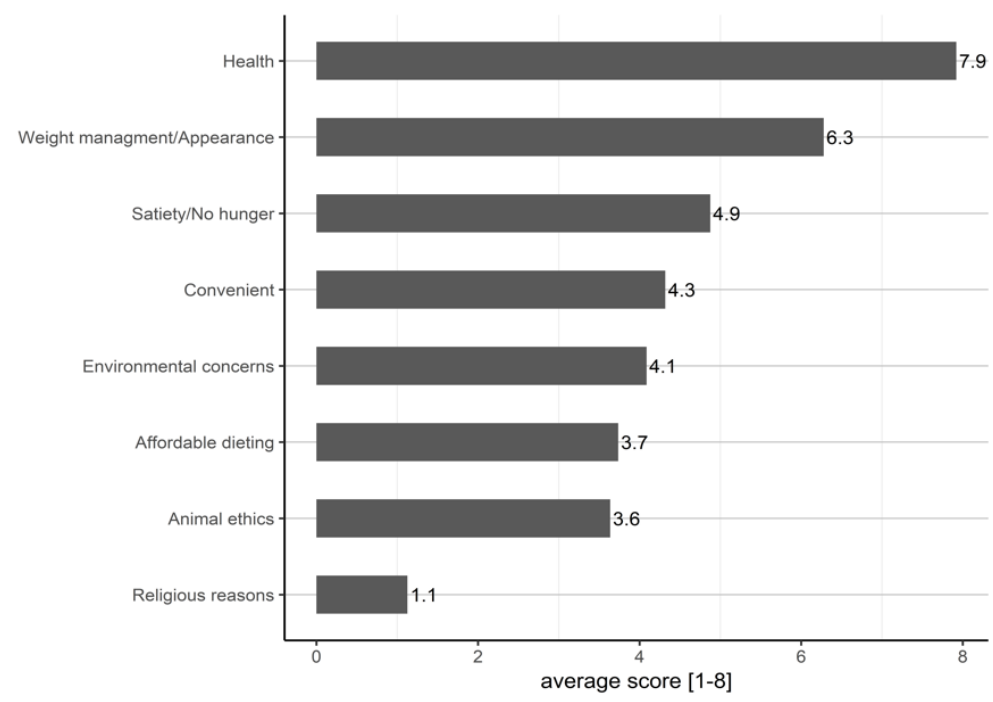

Figure 2: Motives for adopting WFPB dietary pattern. 


\subsection{Anthropometric and Body Composition Measures}

There were no differences in maximal lifetime BM and BMI between the three groups of participants (Table 3; baseline vs. current (after short, medium and long-term time (groups 1, 2, and 3) and combined 0.5-10 years in our program WFPB lifestyle program). At baseline (at the entrance to our WFPB lifestyle program on Western-type diet) there were no differences in BM, BMI and body fat percentage between the three groups of participants. Female groups had statistically significantly different body height, but not baseline body-composition status, while male groups had comparable baseline body height and body composition. Furthermore, female Group 3 had statistically different current BM $(58.4 \pm 6.6 \mathrm{~kg}, \mathrm{p}=0.001)$ than Group $1(68.0 \pm 13.1 \mathrm{~kg})(\mathrm{p}<0.001)$, and, consequently, due to different body height, also absolute MM $(43.0 \pm 3.7 \mathrm{~kg}$ or $74.2 \%$ of BM)) compared to Group 1 (48.1 \pm $6.6 \mathrm{~kg}$ or $70.7 \%$ of $\mathrm{BM})(\mathrm{p}<0.001)$. Therefore, $\mathrm{MMI}$ was a more appropriate tool for monitoring differences in muscle-mass changes and was not shown to be significantly different when we compared the female and male groups. Body-composition changes were favourable for all three groups, for both genders and for the whole sample. There were no differences in BMI, body fat percentage, muscle mass index (MMI) and total body water between the three groups of participants. They improved their baseline mean overweight (pre-obesity) BMI range of $26.4(17.7-50.6) \mathrm{kg} / \mathrm{m}^{2}$ to a normal BMI range of $23.9(17.7-41.4) \mathrm{kg} / \mathrm{m}^{2}$, and decreased BM and BF \% points for $7.1 \pm 8.3 \mathrm{~kg}(\mathrm{p}<$ $0.001)$ and $6.4 \pm 5.6 \%$ points $(\mathrm{p}<0.001)$. 
Table 3: Anthropometric and body composition measures of all participants (0.5-10 years in WFPB lifestyle program) and according to their time in WFPB lifestyle program (short (0.5-<2 years), medium (2-<5 years) and long-term (5-10 years) groups $(1,2$ and 3$))$ and gender.

\begin{tabular}{|c|c|c|c|c|c|c|c|c|}
\hline \multirow{2}{*}{$\begin{array}{c}\text { Parameter } \\
\text { Gender }(\mathrm{F} / \mathrm{M})\end{array}$} & \multirow{2}{*}{$\begin{array}{c}\text { Whole sample }(\mathbf{n}=\mathbf{1 5 1}) \\
\text { Both genders }\end{array}$} & \multicolumn{2}{|c|}{ Group $1(n=51)$} & \multicolumn{2}{|c|}{ Group $2(n=56)$} & \multicolumn{2}{|c|}{ Group $3(n=44)$} & \multirow{2}{*}{$\frac{p \text { - Value }}{F / M}$} \\
\hline & & Female & Male & Female & Male & Female & Male & \\
\hline Height (cm) & $169.0 \pm 8.6$ & $167.7 \pm 7.6$ & $178.5 \pm 5.3$ & $164.2 \pm 5.0$ & $178.8 \pm 4.0$ & $163.5 \pm 4.8$ & $179.8 \pm 6.9$ & $0.009 / \mathrm{NS}$ \\
\hline \multicolumn{9}{|l|}{ Max lifetime } \\
\hline Body mass (kg) & $79.6 \pm 19.7$ & $79.1 \pm 22.6$ & $90.2 \pm 11.5$ & $73.7 \pm 13.7$ & $99.6 \pm 27.4$ & $67.9 \pm 10.5$ & $94.9 \pm 15.5$ & NS \\
\hline $\mathrm{BMI}\left(\mathrm{kg} / \mathrm{m}^{2}\right)$ & $27.7(19.8-54.9)$ & $28.1(19.8-54.9)$ & $28.2(24.1-32.8)$ & $27.4(20.3-39.8)$ & $31.0(23.6-50.6)$ & $25.4(20.4-35.8)$ & $29.4(21.6-37.9)$ & NS \\
\hline \multicolumn{9}{|l|}{ Baseline } \\
\hline Body mass (kg) & $75.8 \pm 18.7$ & $74.8 \pm 20.6$ & $87.2 \pm 11.8$ & $71.1 \pm 14.2$ & $94.4 \pm 27.5$ & $65.4 \pm 9.4$ & $86.3 \pm 16.2$ & NS \\
\hline BMI $\left(\mathrm{kg} / \mathrm{m}^{2}\right)$ & $26.4(17.7-50.6)$ & $26.6(19.1-46.3)$ & $27.3(23.0-32.8)$ & $26.4(17.7-38.3)$ & $29.3(21.8-50.6)$ & $24.5(19.2-32.5)$ & $26.7(19.0-35.3)$ & NS \\
\hline Body fat (\%) & $28.7 \pm 9.2$ & $30.3 \pm 9.6$ & $22.4 \pm 6.0$ & $33.3 \pm 7.9$ & $23.1 \pm 9.4$ & $29.4 \pm 6.7$ & $21.2 \pm 10.0$ & NS \\
\hline \multicolumn{9}{|l|}{ Current } \\
\hline Body mass (kg) & $68.7 \pm 14.0$ & $68.0 \pm 13.1$ & $77.9 \pm 8.2$ & $64.0 \pm 9.6$ & $85.5 \pm 18.8$ & $58.4 \pm 6.6$ & $82.6 \pm 10.2$ & $0.001 / \mathrm{NS}$ \\
\hline $\mathrm{BMI}\left(\mathrm{kg} / \mathrm{m}^{2}\right)$ & $23.9(17.7-41.4)$ & $24.2(18.7-37.3)$ & $24.5(20.8-29.0)$ & $23.8(17.7-30.3)$ & $26.6(22.8-41.4)$ & $21.8(18.3-29.5)$ & $25.5(21.3-26.9)$ & NS \\
\hline Body fat (\%) & $22.3 \pm 7.3$ & $24.4 \pm 8.0$ & $17.1 \pm 6.3$ & $25.8 \pm 6.3$ & $20.0 \pm 5.8$ & $21.9 \pm 5.7$ & $15.3 \pm 5.3$ & NS \\
\hline $\begin{array}{c}\text { Visceral fat } \\
\text { (arbitrary units) }\end{array}$ & $4.1 \pm 3.1$ & $3.5 \pm 2.8$ & $4.3 \pm 3.3$ & $4.0 \pm 2.4$ & $7.2 \pm 4.8$ & $3.1 \pm 2.4$ & $5.1 \pm 3.2$ & NS \\
\hline Muscle mass (kg) & $50.4 \pm 10.1$ & $48.1 \pm 6.6$ & $61.1 \pm 4.8$ & $44.7 \pm 4.6$ & $64.1 \pm 8.2$ & $43.0 \pm 3.7$ & $66.3 \pm 6.6$ & $<0.001 / \mathrm{NS}$ \\
\hline $\begin{array}{l}\text { Muscle mass index } \\
\qquad\left(\mathrm{kg} / \mathrm{m}^{2}\right)\end{array}$ & $17.5 \pm 15.9$ & $17.1 \pm 2.1$ & $19.2 \pm 1.3$ & $16.6 \pm 1.7$ & $20.0 \pm 1.9$ & $17.4 \pm 2.4$ & $20.5 \pm 1.5$ & NS \\
\hline $\begin{array}{l}\text { Total body water } \\
\text { (L) }\end{array}$ & $55.7 \pm 51.6$ & $53.4 \pm 7.4$ & $59.7 \pm 5.3$ & $53.0 \pm 4.7$ & $57.5 \pm 4.1$ & $55.7 \pm 4.3$ & $61.8 \pm 4.1$ & NS \\
\hline
\end{tabular}

Data are mean \pm standard deviation (SD) for normally distributed variables, and median (minimum-maximum) for non-normally distributed ones. 
Detailed BMI and BF \% obesity classification (Table 4) showed that, at the baseline, only 63 females (57.8\%) and 12 males (28.6\%) were within a normal BMI range, whereas the current BMI results showed that the proportion of normal BMI increased by $16.6 \%$ for females and by $26.2 \%$ for males. The improvement toward normal BMI was seen in all group comparisons (Figure 3). BF \% obesity-classification comparison showed similar results (Figure 4). At the baseline, 38 females (34.9\%) and 14 males (33.3\%) were considered as obese but current BF \% status showed improvement to normal $\mathrm{BF} \%$ range by $27.6 \%$ for females and $23.8 \%$ for males. At the end of the study 101 females $(92.7 \%)$ and 38 males (90.5\%) were considered within normal BF \% range. Group comparison showed that Group 1 improved normal BMI from 25 (49\%) to 38 participants (74.5\%), Group 2 from 22 (39.3\%) to 31 participants $(55.4 \%)$, and Group 3 from $28(63.6 \%)$ to 35 participants $(79.5 \%)$. Using BF \% classification, Group 1 improved the proportion of participants with normal BF \% from 35 (68.6\%) to 45 participants (88.2\%), Group 2 from 30 (53.6\%) to 52 participants (92.9\%), and Group 3 from 34 $(77.3 \%)$ to 42 participants (95.5\%). One female was underweight at the entrance to our WFPB lifestyle program, while two females were underweight at present, both with BMI $<18.5$. 
Table 4: Baseline and current BMI and body fat (BF \%) obesity classification of all participants and according to their time in WFPB lifestyle program: short (0.5-<2 years), medium (2-<5 years) and long-term (5-10 years) groups (1, 2 and 3$)$

\begin{tabular}{|c|c|c|c|c|c|c|c|}
\hline \multirow{2}{*}{$\begin{array}{c}\text { Parameter } \\
\text { Gender (F/M) }\end{array}$} & \multicolumn{2}{|c|}{ Whole sample $(\mathrm{n}=151)$} & \multirow{2}{*}{$\frac{p-\text { Value }}{F / M}$} & \multirow[t]{2}{*}{ Group $1(n=51)$} & \multirow[t]{2}{*}{ Group $2(n=56)$} & \multirow[t]{2}{*}{ Group $3(n=44)$} & \multirow{2}{*}{$\frac{\mathbf{p}-\text { Value }}{\mathrm{F} / \mathrm{M}}$} \\
\hline & Female & Male & & & & & \\
\hline Baseline Body mass status, n (\%) & & & 0.005 & & & & NS \\
\hline Underweight (BMI <18.5) & $1(0.9 \%)$ & $0(0.0 \%)$ & & $0(0.0 \%)$ & $1(1.8 \%)$ & $0(0.0 \%)$ & \\
\hline Normal weight (BMI 18.5-24.9) & $63(57.8 \%)$ & $12(28.6 \%)$ & & $25(49.0 \%)$ & $22(39.3 \%)$ & $28(63.6 \%)$ & \\
\hline Pre-obesity (BMI 25-29.9) & $18(16.5 \%)$ & $18(42.9 \%)$ & & $13(25.5 \%)$ & $14(25.0 \%)$ & $9(20.5 \%)$ & \\
\hline Obesity class 1 (BMI 30-34.9) & $20(18.3 \%)$ & $10(23.8 \%)$ & & $8(15.7 \%)$ & $16(28.6 \%)$ & $6(13.6 \%)$ & \\
\hline Obesity class 2 (BMI 35-39.9) & $3(2.8 \%)$ & $1(2.4 \%)$ & & $1(2 \%)$ & $2(3.6 \%)$ & $0(0.0 \%)$ & \\
\hline Obesity class 3 (BMI >40) & $4(3.7 \%)$ & $1(2.4 \%)$ & & $4(7.8 \%)$ & $1(1.8 \%)$ & $0(0.0 \%)$ & \\
\hline BF obesity (\%): & Female: $>35 \%$ & Male: $>25 \%$ & NS & & & & NS \\
\hline No & $71(65.1 \%)$ & $28(66.7 \%)$ & & $35(68.6 \%)$ & $30(53.6 \%)$ & $34(77.3 \%)$ & \\
\hline Yes & $38(34.9 \%)$ & $14(33.3 \%)$ & & $16(31.4 \%)$ & $26(46.4 \%)$ & $10(22.7 \%)$ & \\
\hline Current Body mass status, n (\%) & & & 0.039 & & & & NS \\
\hline Underweight (BMI <18.5) & $2(1.8 \%)$ & $0(0.0 \%)$ & & $0(0.0 \%)$ & $1(1.8 \%)$ & $0(0.0 \%)$ & \\
\hline Normal weight (BMI 18.5-24.9) & $81(74.3 \%)$ & $23(54.8 \%)$ & & $38(74.5 \%)$ & $31(55.4 \%)$ & $35(79.5 \%)$ & \\
\hline Pre-obesity (BMI 25-29.9) & $20(1.8 \%)$ & $16(38.1 \%)$ & & $9(17.6 \%)$ & $19(33.9 \%)$ & $9(20.5 \%)$ & \\
\hline Obesity class 1 (BMI 30-34.9) & $4(3.7 \%)$ & $2(4.8 \%)$ & & $2(3.9 \%)$ & $3(5.4 \%)$ & $1(2.3 \%)$ & \\
\hline Obesity class 2 (BMI 35-39.9) & $2(1.8 \%)$ & $0(0.0 \%)$ & & $2(3.9 \%)$ & $0(0.0 \%)$ & $0(0.0 \%)$ & \\
\hline Obesity class 3 (BMI >40) & $0(0.0 \%)$ & $1(2.4 \%)$ & & $0(0.0 \%)$ & $1(1.8 \%)$ & $0(0.0 \%)$ & \\
\hline BF obesity (\%): & Female: $>35 \%$ & Male: $>25 \%$ & NS & & & & NS \\
\hline No & $101(92.7 \%)$ & $38(90.5 \%)$ & & $45(88.2 \%)$ & $52(92.9 \%)$ & $42(95.5 \%)$ & \\
\hline Yes & $8(7.3 \%)$ & $20(9.5 \%)$ & & $6(11.8 \%)$ & $4(7.1 \%)$ & $2(4.5 \%)$ & \\
\hline
\end{tabular}

Data are mean \pm standard deviation (SD) for normally distributed variables. BMI classifications by the World Health Organization [69], BF \% obesity classification by WHO [70]. 
Figure 3: Group comparison of obesity by normal BMI (classifications by WHO [69])

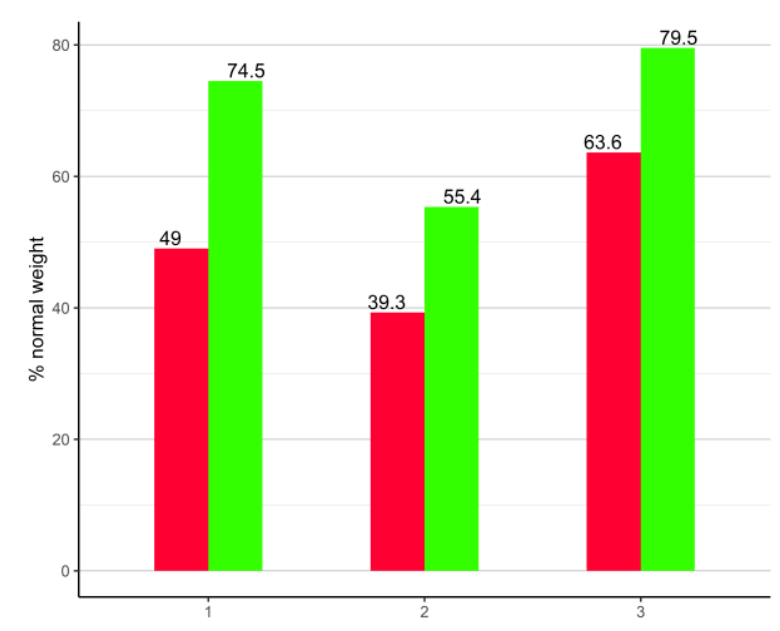

Figure 4: Group comparison of obesity by body fat (\%) (classification by WHO (WHO, 1995)

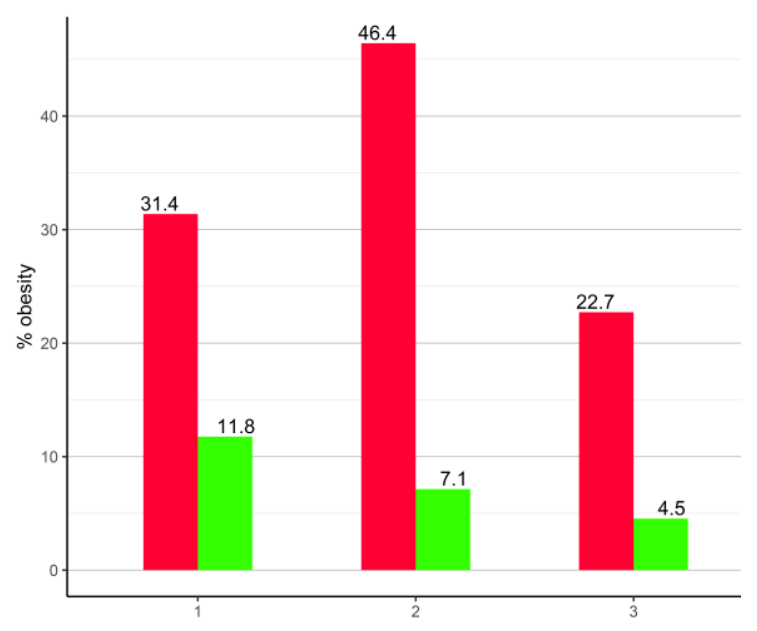

Baseline vs. current comparison of BMI, BM, BF \% according to BMI classification (Table 5) showed that all participants $(\mathrm{n}=151)$ significantly improved their obesity indices. They experienced a mean BMI reduction of $-2.5 \pm 2.8 \mathrm{~kg} / \mathrm{m}^{2}$ (from baseline mean pre-obesity BMI range $\left(26.4 \pm 5.6 \mathrm{~kg} / \mathrm{m}^{2}\right.$ ) to normal BMI $\left(23.9 \pm 3.8 \mathrm{~kg} / \mathrm{m}^{2}\right),(\mathrm{p}<0.001)$. They also achieved BM reduction of $7.1 \pm \mathrm{SD} 5.4 \mathrm{~kg}(\mathrm{p}<$ 0.001 ) and body fat percentage reduction of $6.4 \pm 5.6 \%$ points $(p<0.001)$. Participants who had the highest $\mathrm{BMI}$ at baseline (BMI $\geq 30->40$; obesity classes 1, 2 and 3) lost the most of BMI units, total BM and body fat percentage. BMI units $\left(-5.6 \pm\right.$ SD $2.9 \mathrm{~kg} / \mathrm{m}^{2},-2.4 \pm 1.8 \mathrm{~kg} / \mathrm{m}^{2}$ and $\left.-0.9 \pm 1.5 \mathrm{~kg} / \mathrm{m}^{2}\right)$, total $\mathrm{BM}(-16.1 \pm \mathrm{SD} 8.8 \mathrm{~kg},-7.1 \pm 5.4 \mathrm{~kg}$ and $-2.5 \pm 4.5 \mathrm{~kg})$ and body fat percentage points $(-9.5 \pm \mathrm{SD} 5.7 \%,-$ $6.6 \pm 4.6 \%$ and $-4.7 \pm 5.3 \%)$ for participants who had baseline BMI in obese $(n=39)$, overweight $(n=36)$ and normal range $(n=75)$, respectively ( $p$ baseline vs. current $<0.001$ for all). 


\section{Discussion}

Evaluation of the long-term (0.5-10 years) effect of WFPB lifestyle program on lifestyle and BM management demonstrated statistically significant BM loss, decrease in BMI as well as BF percentage points in all three groups. We hypothesized that adopting WFPB lifestyle program for 0.5$<2$ years was not yet adequate for final and sustainable beneficial changes, but proved the opposite. There were no significant differences in relative body-composition changes (BMI, BF \% and MMI) in the group comparison, and therefore our hypothesis was rejected. According to the results of the study, most significant and long-lasting changes occurred already within the first two years in WFPB lifestyle program. The new, healthy lifestyle and WFPB dietary pattern were transferred further to $85.6 \%$ of underaged children of participants.

\subsection{Motives for PBD}

In our study we found that a major motive for WFPB dietary pattern was the health-benefit aspect (score 7.9 of 8 ), which was followed by BM management/appearance (6.3), eating to satiety/no hunger (4.9), convenience (4.3), environmental concerns (4.1), affordable overall dieting (3.7), animal ethics (3.6), and religious reasons (1.1) $(90 \%$ had chosen this as the least important motive of adopting PBD). The health-benefit aspect was chosen as the primary motive to start eating PBD by $93 \%$ of the participants, while $93 \%$ of the sample chose religion to be the least important motive for following a PBD. We did not find unhealthy or obsessive eating behaviour associated with PBD.

According to previous studies, frequently emerging motives for choosing a strict PB (vegan) diet included ethical (concerns about animal welfare, suffering, farming, rights, and speciesism), health-related benefits (concerns about various chronic diseases risk, overall health and longevity/mortality), BM management (concerns about being pre-obese or obese), environmental (concerns about long-term ecological sustainability of human food production, lower levels of greenhouse-gas emissions, and other planet-health aspects), and religious reasons (part of religious lifestyle rules) [71-74]. A study on 100 self-reported vegans (defined by not eating meat, fish, poultry, but eating dairy or egg-containing products less than once per month) that were living in different U.S. states and recruited via print and electronic advertising showed that $47 \%$ of the vegans reported health benefits as their main reason for being vegan, while animal-welfare, religious, and environmental beliefs were identified by $40 \%, 9 \%$, and $2 \%$, respectively. In contrast, another study recruited an international sample of 246 vegans through events and social media targeting vegans found that ethical reasons (201) were almost fivefold more the cited reason for adopting PBD compared to health benefits (45) [72]. A European study conducted in seven vegan supermarkets in Germany on 329 consumers following the vegan diet revealed three main motives: animal-related motives (89.7\% respondents), motives related to personal well-being and/or health $(69.3 \%)$, and environment-related motives $(46.8 \%) ; 81.9 \%$ of respondents mentioned more than one motive. The authors concluded that making a dichotomous segmentation into ethical versus self-oriented consumers disregards the fact that many consumers following a vegan diet are driven by more than one motive [73]. Other authors further explored the question whether there were possible differences in conviction, nutrition knowledge, dietary restriction, and duration of adherence to vegetarianism. According to their original reasons for becoming vegetarian, 292 individuals (125 vegans and 109 vegetarians) were grouped (58 health and 234 ethical). The authors' results suggested that ethical vegetarians could experience stronger feelings of conviction and consume fewer animal products than health vegetarians, and may remain vegetarians for longer. Nutrition knowledge did not differ 
between the two groups [75]. Furthermore, it has been hypothesized, especially in older observational studies, that there is an association between plant-based diets and/or meat avoidance, and more eating disorders (ED) [76]. Several recent studies suggest that a vegan diet with various dietary restrictions does not directly lead to an eating disorder, and that cause and effect cannot be determined [77]. However, studies suggest that a vegan diet, contrary to common belief, is not associated even with a rather new form of a yet-unclassified eating disorder that is an obsession of healthy eating (orthorexic eating behaviour) [78]. Vegans and omnivores do not markedly differ in reported unhealthy eating attitudes and behaviours, and when they do, vegans appear to endorse overall healthier attitudes and behaviours toward food $[1,76,79,80]$.

\subsection{Lifestyle factors}

At the baseline, when entering into our WFPB lifestyle program, the participants were differently physical active, on average more sedentary without organized or systematic PA, more participants smoked and more cigarettes and many of them had increased CVD risk factors, but we did not systematically record them. Participants in our WFPB lifestyle program implemented the proposed lifestyle changes very well and had on average a very limited daily sitting time, with PA above the recommended, and had the recommended sleep quality and low perceived stress level. Centenarian and longest-lived-population studies associate the low incidence and mortality of cancer and CVD with exclusive or predominant PBD [21,81] but also with their lifestyle behaviours [22,39,71]. Often, it is assumed that PBD health benefits compared to an omnivore diet are more related to plantbased dieters accompanying it with healthier lifestyle choices. Lifestyle factors such as regular PA, sleep quality, stress management, and socialization [36-39] were recognized to play an important role in health, disease prevention, and longevity. Two large (UK and U.S.) cross-sectional studies on PB dieters found that participants had lower incidence of smoking, consumed less alcohol and less frequently, were physically more active, and were less sedentary [21,22]. PB dietary intervention alone or accompanied by other lifestyle changes has demonstrated impressive health benefits. For example, three randomized control trials showed major CVD (Lifestyle Heart Trial) (Ornish et al., 1990, 1998) and early, low-grade prostate-cancer reversal in men (Prostate Cancer Lifestyle Trial) [84] where researchers predominately used PBD as intervention, supplemented with soy isolate proteins, several dietary supplements, and fortified conventional foods, and also included large overall lifestyle changes (PA, stress management, smoking cessation, and group psychosocial support). On the other hand, other researchers only used long-term WFPB diet as intervention without [85] and with a control group [86], without systematically interfering in other lifestyle components (though are accompanying almost automatically); they also measured remarkable results with CVD reversal.

Results in our study confirmed that a long-term WFPB lifestyle program that is primary based on Supp WFPB diet and PA has a very beneficial, long-lasting impact on healthy BM management and improved body-composition. Interestingly, we found favourable average weekly (five hours per day) and weekend sitting (four hours per day) status compared to sedentary Western-type lifestyle trends [87]. PA or a low level of PA was found to be the $10^{\text {th }}$ leading risk factor from the list of burden of diseases attributable to risk factors [88], while every day of prolonged sitting was found to potentially have a causal relationship between sedentary behaviour and all-cause mortality based on several epidemiological criteria [89]. Furthermore, regular higher PA may not neutralize all the negative health effects of every-day occupational sitting [90]. The time spent sitting was in one study independently associated with total mortality regardless of PA level. The combination of both sitting and being less physically active was associated in this study with up to $94 \%$ and $48 \%$ increase in all- 
cause death rates in women and men compared with those who reported sitting the least and being most active [91]. Since studies showed that PA alone has little or no significant long-term relationship on BM or body composition [43,92,93], we could assume that this is true also for PB dieters. PA or exercise was shown to have an important role in BM management, particularly when combined with dietary changes [41,42]. Researchers also suggested that PA might not work as expected in BMmanagement studies because of the irregularity of performing PA or exercise and the concomitant increase in energy intake in PA and BM-loss or body-composition studies [94].

WFPB dieters in our study incorporated PA more as an integrated lifestyle component and in combination with a diet and where commitment is maintained through the assistance of an extensive support system and community networks. People in this kind of environment became more aware of the importance of being physically active, and stayed inspired by the health and life-quality benefits and were also encouraged by other individuals on this path of change when they could see results on their body composition or/and health. Outdoor habitual PA or PA in nature (biking, hiking, or regular walking) for our studied participants became a norm, rhythm, and positive "addiction" that could then be called a lifestyle choice. The reason for our high PA results, besides the possible overreporting of estimated PA, might lie in physically active lifestyle (walking on diverse hiking surfaces) and some form of strength training (using own BM or attending fitness gyms) is promoted as an integral part of PBD lifestyle. Another reason for high PA may be because the study was performed during the summertime that is known to be the most physically active period in the year, and when they encourage each other to do some PA for themselves, they socialize more, and are more healthoriented. In our study, we used a self-administered L-IPAQ questionnaire with acceptable reliability and validity when assessing levels and patterns of PA in healthy adults in diverse settings [55,95,96], and also for both genders of older adults (over 65 years of age) [97], which was important for our sample in some cases. The L-IPAQ method is highly reliable; an assessment of test-retest repeatability produced a correlation of 0.8 [95]. On average, our participants spent 5542 METs minutes weekly (792 METs minutes per day), where the most METs came from moderate PA, followed by vigorous PA and daily walking. The frequency of organized resistance/weight-lifting workouts that lasted at least 30 minutes was estimated as average to 2.7 times weekly per participants. Some authors suggested that a PA level of 3000 to 4000 METs minutes weekly is where most health gains occur [98].

Participants in our study reported a low perceived stress level (PSQ $=0.3 \pm 0.1$ ) with a favourable positive-item score $(17.3 \pm 3.9)$ and no significant difference between the groups. Our results on perceived-stress status are consistent with the results of the first study investigating selfreported mental-health domains in a large sample of vegans $(n=109)$ with vegetarians $(n=283)$ and omnivores $(n=228)$ [99]. In the last few decades, society has also been faced with other important lifestyle-behaviour challenges, such as stress and sleeping quality. Stress exposure increases the risk for poor clinical outcomes across a variety of major health conditions [38], while sleeping disorders have vast potential for adverse short- and long-term health consequences in otherwise healthy individuals, as well as those with underlying medical conditions [100]. Researchers found that vegans reported less anxiety and stress. In recent prospective study and their previous cross-sectional and pilot randomized controlled studies [101,102], striving to eat a more plant-based diet with lower intake of animal food was associated with better mood and mental health in general [9].

Concerning sleep, we found that the average time to go to sleep was mostly late (22:46), average wake-up time is at 5:54 am, people fall asleep in less than 15 minutes (11.9 minutes), and sleep 
duration is less than 7 hours per night (6.97 hours). Dietary composition has been shown to influence sleep duration, quality, and behaviours [99,103]. It was suggested that increasing fruit and vegetable intake, choosing whole grains (high in fibre), milk products, fish, and vegetable oils (low in saturated fat) also showed sleep-promoting effects [99]. A recent review of epidemiological studies showed that a PBD may provide additional benefits to health via its potential effect on sleep quality, but failed to establish a causal relationship between a plant-rich dietary pattern and sleep health [104]. Findings from countries across Asia and Africa [105] and European countries [106] showed that sleep disturbance was associated with socioeconomical insecurity and other environmental $24 \mathrm{~h}$ lifestyle factors; thus, sleeping disorders are an emerging public-health issue.

Other lifestyle behaviours that are important and were included in our study are drinking alcohol and smoking tobacco products. PB dieters are known to drink less alcohol compared to the general population. In our study, we found that only $3(2 \%)$ participants stated alcohol consumption (less than $0.5 \mathrm{~g}$ of alcohol per day for the whole sample). Furthermore, 114 participants reported to not smoke ever (76\%), 9 participants reported to still smoke (6\%) but less than before adopting Supp WFPBD, 26 participants stopped smoking when they adopted the Supp WFPBD (17\%; 5 participants had been smoking for over 20 years, 13 for over 10 years, and 24 for more than 5 years), and 1 participant did not want to report their smoking status. Since the studied population were active members of society, meaning studying, working, having families, and being physically active, these results might not be surprising. Overall, the study results clearly showed that it is possible to maintain a favourable balance between compliance to Supp WFPBD, PA, and healthy lifestyle factors in a modern, fast-paced lifestyle. In a Swiss study, researchers found that alcohol abstinence was more prevalent among vegans (28\%) compared to vegetarians $(6.1 \%)$ or omnivores $(3.2 \%)$ [107].

\subsection{Anthropometric and body composition measures}

At the baseline, when entering into our WFPB lifestyle program, participants were not highly motivated or had preference toward WFPB dietary pattern, what is evident from their average maximal BM and baseline anthropometric and body composition status. Due to the BMI limitations, we combined in our study the BMI with body-composition profile measurements, which is especially important for smaller-scale observational studies and for people with sarcopenic obesity, as was suggested by some researchers in this field $[108,109]$. Our results of body-composition status of the whole sample and of the three compared groups (divided by gender) showed an improvement in adopting the WFPB lifestyle. The possible reasons for the favourable outcome may be due to quality and quantity of Supp WFPBD with the combination of being physically active and other lifestylerelated factors (good sleep, lower stress, limited alcohol intake, and being/becoming a non-smoker or limiting smoking).

It is widely accepted that body composition can independently influence health [110]. BMI has been consensually accepted, at least in epidemiological research, as a practical marker adopted as a surrogate for obesity that is associated with a risk for chronic diseases and an increase in all-cause mortality [109]. However, BMI is a poor index for total fat or fat distribution [111], and was criticized for its lack of sensitivity to distinguish BM between fat mass and lean mass [109,112]. In brief, the BMI from one review was shown to not be sensitive enough for up to $50 \%$ individuals with increased body fat at normal BMI [113]. 
Other studies on PB dieters found large variety in BMI status, and we could not directly compare our study results with other cross-sectional studies since they did not follow the sample in the transition from the end of a Western-type diet (in a pre-obesity state) through the transitional period to a PBD lifestyle, and since many of them did not divide the samples by gender (see below). Nevertheless, the average BMI of vegans in the Epic Oxford study was $22.49 \mathrm{~kg} / \mathrm{m}^{2}$ for females and $21.98 \mathrm{~kg} / \mathrm{m}^{2}$ for males [114], while in Adventist Health Study 2, 5548 vegans were found to have 24.1 $\mathrm{kg} / \mathrm{m}^{2} \mathrm{BMI}$ [21], which is interesting since the Epic Oxford vegans consumed slightly more calories, a lot more total fat, and a lot less dietary fibre. The lower BMI of Epic Oxford vegans compared to the Adventist Health Study 2 vegans cannot be attributed to the possible differences in female and male ratios (females in general have higher BMI) in the studied populations since both studied samples had similar ratios of female and male participants, namely, $72 \%$ more females than males in the Epic Oxford study [114], and 75\% more females in the Adventist Health study 2 [21]. PA may account for more of the differences in the compared BMI samples, but differences in PA for which we do not have comparable data are unlikely to explain the higher BMI of UK vegans, since studies showed that PA alone has little long-term relationship on BM or body composition $[43,92,93]$. We may assume that they lied about the reason in their baseline status and the motive of adopting PBD, meaning that the UK sample may have started PBD lifestyle in a better baseline body composition than the U.S. sample, but we cannot make a solid conclusion. Similarly unexpectedly, researchers measured the BMI of British Indian vegans and found that their BMI was just below pre-obesity level $\left(24.8 \mathrm{~kg} / \mathrm{m}^{2}\right)$ but with a high body-fat percentage (32.7\%) [115]. The body composition of low-calorie and low-protein 21 U.S. raw food vegans was determined by DXA, whereas their BMI was $21.3 \mathrm{~kg} / \mathrm{m}^{2}$ and body-fat percentage was $13.7 \%$ for men and $26.9 \%$ for women [116]. BMI was also found to be very similar when compared with 75 Danish vegans, measured by BIA, where researchers found their average to be 21 $\mathrm{kg} / \mathrm{m}^{2}$ [117] and Swiss vegans $\left(21.6 \mathrm{~kg} / \mathrm{m}^{2}\right)$, but we do not know the gender differences [107], which is big limitation for a serious comparison with our results. An Italian study found that the average BMI of 26 vegans ( 9 men and 17 women) was $23.7 \mathrm{~kg} / \mathrm{m}^{2}$ and, with the BIA method, body-fat percentage was $25.6 \%$ [118], but the researchers did not divide body-fat percentage by gender. Another European cohort study (Epic Norfolk) that included only a small sample of vegans (12 males and 16 females) found that vegans had BMI in pre-obesity range with 28 and $27 \mathrm{~kg} / \mathrm{m}^{2}$ for males and females [119]. Meta-analysis of 37 studies on 12,241 vegans showed that BMI was on average within the healthy weight range, but there was no difference in BMI for vegans compared to controls in Asia $\left(23.3 \mathrm{~kg} / \mathrm{m}^{2}\right)$ but, for non-Asian studies, the difference was $-1.92 \mathrm{~kg} / \mathrm{m}^{2}$ [120].

\subsection{Participants' children}

In our study, we found that the new, healthy WFPB lifestyle was transferred further to $85.6 \%$ of children aged $<18$ years and to $28.2 \%$ of children aged $>18$ years, showing that it was more than three times more likely to be transferred to under-aged children than to adult children. Such high transfer of WFPB dietary pattern in Slovenia is of special success, as in Slovenia families and children are not encouraged to practice a WFPB diet, as it has been the case in US (for 10 years already) [1,121], Australia [5], Canada [4], Portugal [6], Great Britain [3] and Italy [2]. In California for example, schools receive incentives to support more plant-based meals [122], similarly in Brazil [123]. In Europe, Portugal passed a new law with vegan menu option requirement [124].

\section{Strengths and Limitations}


The strength of our study includes its long-term nature since the participants were on our WFPB lifestyle program from 0.5-10 years. We followed their entire transformation from a Westerntype, more sedentary lifestyle and anthropometric measures and their baseline body-composition components. A unique feature of our study is describing the sample in detail for both genders, including their lifestyle and accompanying extensive support system. The study has also a good geographical representation since our participants lived dispersed across the country, in six regions in Slovenia, in urban, suburban, and rural areas. All measurements of quite a large sample were addressed within a short period of 14 days. The investigated WFPB lifestyle program was already confirmed in two of our previous intervention-type studies to be effective for BM normalization as well as the short and medium term management of several CVD risk factors [28,44]. This study confirmed the previously shown health benefits to have a long-lasting effect. It is also the first crosssectional study on Supp WFPB dieters that assessed several other important data (Table 1), such as how many children $(<18$ years and $>18$ years) were born to participants practicing WFPB lifestyle, their infants' birth BM, and pregnancy BM gain. Most importantly, we documented an extremely high successful transfer of healthy life-style to their children (to $85.6 \%$ of children aged $<18$ years).

We are aware that our study has some obvious limitations inherent to epidemiological studies, and due to the possibility of under- or over-reported estimates of lifestyle components that were assessed by questionnaires. As a limitation, we see a constant need for a larger cross-sectional sample (especially of males) and for performing a non-randomized study. We assumed that, we might have lost power of the male sample, since it is known from WFPB dietary-intervention studies that the ratio of women to men in the sample is strongly in favour of women. In addition, we could not exclude the possible unknown impact of people who were within the set criteria, but did not respond or were not willing to participate in the study. Furthermore, the use of a medically-approved bioimpedance body-composition monitor (our only option to involve this number of WFPB dieters to participate in study during the summertime) instead of more accurate methods, for example, DEXA, was another limitation. Additionally, our results were not limited to diet only, since participants were practising a healthy and active lifestyle, where regular PA is associated with lower BMI and BF percentage [125], especially in combination with a weight-management dietary program [41]. Additional stimulus for the better success of Supp WFPB diet was the enhanced and extensive support system [28], since behaviour changes [126] and motives related to personal well-being and health [75] may be a crucial part to remain a plant-based dieter for the long term. In the future, it would be valuable to investigate the reasons why most of our screened people that adopted plant-rich diet did not adopt a strict Supp WFPB diet or exclusively WFPBD.

\section{Conclusion}

To the best of our knowledge, this is the first study to assess the effects of a short -, medium-, and long-term (0.5-10 years) WFPB lifestyle program on body composition indices compared to baseline characteristics. We confirmed the benefits of ad libitum Supp WFPB dietary pattern, without calorie counting or portion control, associated with accompanying healthier lifestyle choices. The study extended this finding by discovering no differences in lifestyle and body composition indices between the participants of our WFPB lifestyle program for a short-, medium- and long-term. Our data show that the majority of beneficial effects can be achieved already within the first two years, and successfully maintained in the long term (i.e., 10 years) through an extensive support system. Our results are of an utmost importance, contrasting the majority of weight-loss programs which have a 
high failure rate within the first two years and in the long-run. The present study confirms the benefits of WFPB lifestyle program, including very effective and sustainable weight loss, the reduction of BF percentage and handing over healthy and active lifestyle to the next generation (85.6\% of underage children) in a real world setting with participants responsible for their financially sustainable eating and own food preparation.

Author contributions: Conceptualization, B.J. and N.F.M.; Applied for and Ethics Committee approval, B.J., N.F.M. and S.P., Recruited the participants, B.J., B.J. (Barbara Jakše) and S.P., Resources, N.F.M, B.J. and B.J. (Barbara Jakše) N.F.M.; Writing - Original Draft Preparation, B.J. and N.F.M.; Writing - Review \& Editing, B.J., N.F.M., B.J. (Barbara Jakše), S.P., J.P.; Visualization, B.J., N.F.M. and J.P.; Supervision, N.F.M, S.P. and J.P.; Project Administration, N.F.M. and B.J. (Barbara Jakše); Funding Acquisition, N.F.M. and B.J. (Barbara Jakše). All authors had full access to data and revised and approved the manuscript for publication. The guarantor; B.J., N.F.M., S.P. and J.P.

Acknowledgments: The authors wish to thank all health coaches for their collaboration. We would like to thank Maja Lampe (M.S. students at the University of Ljubljana, Biotechnical Faculty) for electronic questionnaire input, to Uroš Godnov (Faculty of Management, University of Primorska) for his assistance with the statistical analysis and Alenka Polajnar Gantar for language review. Last, but not least, we would like to show our gratitude to all participants in the study. This work would not have been possible without them.

Funding: The work was partly financially supported by the Slovenian Research Agency (Research Program P30395: Nutrition and Public Health) and partly by resource of Barbara Jakše, sole proprietor.

Conflicts of Interest: J.P. and N.F.M. do not declare any conflict of interest and collaborated as independent researchers. B.J. (Barbara Jakše) and S.P. are receiving royalty compensation at Herbalife Nutrition, which did not have any role in the design of the study, collection, analysis, and interpretation of data nor in writing the manuscript. B.J. (Boštjan Jakše) is B.J. (Barbara Jakše)'s spouse and therefore derives income from the same sources.

\section{References}

1. Melina, V.; Craig, W.; Levin, S. Position of the Academy of Nutrition and Dietetics: Vegetarian Diets. J. Acad. Nutr. Diet. 2016, 116, 1970-1980.

2. Agnoli, C.; Baroni, L.; Bertini, I.; Ciappellano, S.; Fabbri, A.; Papa, M.; Pellegrini, N.; Sbarbati, R.; Scarino, M.L.; Siani, V.; et al. Position paper on vegetarian diets from the working group of the Italian Society of Human Nutrition. Nutr. Metab. Cardiovasc. Dis. 2017, 27, 1037-1052.

3. BDA British Dietetic Association confirms well-planned vegan diets can support healthy living in people of all ages Available online: https://www.bda.uk.com/news/view?id=179 (accessed on Oct. 22, 2019).

4. Dietitians of Canada What You Need to Know About Following a Vegan Eating Plan - Unlock Food Available online: https://www.unlockfood.ca/en/Articles/Vegetarian-and-VeganDiets/What-You-Need-to-Know-About-Following-a-Vegan-Eati.aspx (accessed on Oct 22, 2019).

5. ADG Australian Dietary Guidelines I NHMRC Available online: https://www.nhmrc.gov.au/about-us/publications/australian-dietary-guidelines (accessed on Oct 22, 2019).

6. Gomes, S.C.; João, S.; Pinho, P.; Borges, C.; Santos, C.T.; Santos, A.; Design, P.G.; Mendes De Sousa Editor, S. National Programme for the Promotion of Healthy Eating Guidelines for a Healthy Vegetarian Diet; 2015; ISBN 978-972-675-228-8. 
7. Anderson, L. U.S. Doctors Blast Belgian Misinformation on Vegan Diets Available online: https://www.pcrm.org/news/news-releases/us-doctors-blast-belgian-misinformation-vegandiets (accessed onOct 22, 2019).

8. Gili, R. V.; Leeson, S.; Montes-Chañi, E.M.; Xutuc, D.; Contreras-Guillén, I.A.; Guerrero-Flores, G.N.; Martins, M.C.T.; Pacheco, F.J.; Pacheco, S.O.S. Healthy Lifestyle Practices among Argentinian Vegetarians and Non-Vegetarians. Nutrients 2019, 11.

9. Beezhold, B.; Radnitz, C.; Rinne, A.; DiMatteo, J. Vegans report less stress and anxiety than omnivores. Nutr. Neurosci. 2015, 18, 289-296.

10. Le, L.; Sabaté, J.; Singh, P.; Jaceldo-Siegl, K. The Design, Development and Evaluation of the Vegetarian Lifestyle Index on Dietary Patterns among Vegetarians and Non-Vegetarians. Nutrients 2018, 10, 542.

11. Crowe, F.L.; Appleby, P.N.; Travis, R.C.; Key, T.J. Risk of hospitalization or death from ischemic heart disease among British vegetarians and nonvegetarians: results from the EPICOxford cohort study. Am. J. Clin. Nutr. 2013, 97, 597-603.

12. Satija, A.; Hu, F.B. Plant-based diets and cardiovascular health. Trends Cardiovasc. Med. 2018, $28,437-441$.

13. Dinu, M.; Abbate, R.; Gensini, G.F.; Casini, A.; Sofi, F. Vegetarian, vegan diets and multiple health outcomes: A systematic review with meta-analysis of observational studies. Crit. Rev. Food Sci. Nutr. 2017, 57, 3640-3649.

14. Kahleova, H.; Levin, S.; Barnard, N. Cardio-Metabolic Benefits of Plant-Based Diets. Nutrients 2017, 9, 848 .

15. McMacken, M.; Shah, S. A plant-based diet for the prevention and treatment of type 2 diabetes. J. Geriatr. Cardiol. 2017, 14, 342-354.

16. Viguiliouk, E.; Kendall, C.W.; Kahleová, H.; Rahelić, D.; Salas-Salvadó, J.; Choo, V.L.; Mejia, S.B.; Stewart, S.E.; Leiter, L.A.; Jenkins, D.J.; et al. Effect of vegetarian dietary patterns on cardiometabolic risk factors in diabetes: A systematic review and meta-analysis of randomized controlled trials. Clin. Nutr. 2019, 38, 1133-1145.

17. Satija, A.; Bhupathiraju, S.N.; Rimm, E.B.; Spiegelman, D.; Chiuve, S.E.; Borgi, L.; Willett, W.C.; Manson, J.E.; Sun, Q.; Hu, F.B. Plant-Based Dietary Patterns and Incidence of Type 2 Diabetes in US Men and Women: Results from Three Prospective Cohort Studies. PLOS Med. 2016, 13, e1002039.

18. Qian, F.; Liu, G.; Hu, F.B.; Bhupathiraju, S.N.; Sun, Q. Association Between Plant-Based Dietary Patterns and Risk of Type 2 Diabetes. JAMA Intern. Med. 2019.

19. Barnard, N.D.; Levin, S.M.; Yokoyama, Y. A Systematic Review and Meta-Analysis of Changes in Body Weight in Clinical Trials of Vegetarian Diets. J. Acad. Nutr. Diet. 2015, 115, 954-969.

20. Turner-McGrievy, G.; Mandes, T.; Crimarco, A. A plant-based diet for overweight and obesity prevention and treatment. J. Geriatr. Cardiol. 2017, 14, 369-374.

21. Orlich, M.J.; Singh, P.N.; Sabaté, J.; Jaceldo-Siegl, K.; Fan, J.; Knutsen, S.; Beeson, W.L.; Fraser, G.E. Vegetarian Dietary Patterns and Mortality in Adventist Health Study 2. JAMA Intern. Med. 2013, 173, 1230.

22. Davey, G.K.; Spencer, E.A.; Appleby, P.N.; Allen, N.E.; Knox, K.H.; Key, T.J. EPICOxford:lifestyle characteristics and nutrient intakes in a cohort of 33883 meat-eaters and 31546 
non meat-eaters in the UK. Public Health Nutr. 2003, 6, 259-268.

23. Clarys, P.; Deliens, T.; Huybrechts, I.; Deriemaeker, P.; Vanaelst, B.; De Keyzer, W.; Hebbelinck, M.; Mullie, P. Comparison of nutritional quality of the vegan, vegetarian, semi-vegetarian, pesco-vegetarian and omnivorous diet. Nutrients 2014, 6, 1318-32.

24. Fildes, A.; Charlton, J.; Rudisill, C.; Littlejohns, P.; Prevost, A.T.; Gulliford, M.C. Probability of an Obese Person Attaining Normal Body Weight: Cohort Study Using Electronic Health Records. Am. J. Public Health 2015, 105, e54-9.

25. Wing, R.R.; Phelan, S. Long-term weight loss maintenance. Am. J. Clin. Nutr. 2005, 82, 222S$225 S$.

26. Anderson, J.W.; Konz, E.C.; Frederich, R.C.; Wood, C.L. Long-term weight-loss maintenance: a meta-analysis of US studies. Am. J. Clin. Nutr. 2001, 74, 579-584.

27. Moore, W.J.; McGrievy, M.E.; Turner-McGrievy, G.M. Dietary adherence and acceptability of five different diets, including vegan and vegetarian diets, for weight loss: The New DIETs study. Eat. Behav. 2015, 19, 33-38.

28. Jakše, B.; Jakše, B.; Pajek, J.; Pajek, M. Effects of ad libitum consumed, low-fat, high-fiber plantbased diet supplemented with plant-based meal replacements on cardiovascular risk factors. Food Nutr. Res. 2019, 63.

29. Kent, L.; Morton, D.; Rankin, P.; Ward, E.; Grant, R.; Gobble, J.; Diehl, H. The effect of a low-fat, plant-based lifestyle intervention (CHIP) on serum HDL levels and the implications for metabolic syndrome status - a cohort study. Nutr. Metab. (Lond). 2013, 10, 58.

30. Mishra, S.; Xu, J.; Agarwal, U.; Gonzales, J.; Levin, S.; Barnard, N.D. A multicenter randomized controlled trial of a plant-based nutrition program to reduce body weight and cardiovascular risk in the corporate setting: the GEICO study. Eur. J. Clin. Nutr. 2013, 67, 718-724.

31. Dunn-Emke, S.R.; Weidner, G.; Pettengill, E.B.; Marlin, R.O.; Chi, C.; Ornish, D.M. Nutrient Adequacy of a Very Low-Fat Vegan Diet. J. Am. Diet. Assoc. 2005, 105, 1442-1446.

32. Campbell, E.K.; Fidahusain, M.; Campbell II, T.M. Evaluation of an Eight-Week Whole-Food Plant-Based Lifestyle Modification Program. Nutrients 2019, 11, 2068.

33. WHO The WHO cross-national study of health behavior in school-aged children from 35 countries: findings from 2001-2002. J. Sch. Health 2004, 74, 204-6.

34. Li, Y.; Pan, A.; Wang, D.D.; Liu, X.; Dhana, K.; Franco, O.H.; Kaptoge, S.; Di Angelantonio, E.; Stampfer, M.; Willett, W.C.; et al. Impact of Healthy Lifestyle Factors on Life Expectancies in the US Population. Circulation 2018, 138, 345-355.

35. Larsson, S.C.; Kaluza, J.; Wolk, A. Combined impact of healthy lifestyle factors on lifespan: two prospective cohorts. J. Intern. Med. 2017, 282, 209-219.

36. Warburton, D.E.R.; Bredin, S.S.D. Health benefits of physical activity. Curr. Opin. Cardiol. 2017, $32,541-556$.

37. Grandner, M.A.; Alfonso-Miller, P.; Fernandez-Mendoza, J.; Shetty, S.; Shenoy, S.; Combs, D. Sleep. Curr. Opin. Cardiol. 2016, 31, 551-565.

38. Slavich, G.M. Life Stress and Health: A Review of Conceptual Issues and Recent Findings. Teach. Psychol. 2016, 43, 346-355.

39. Buettner, D.; Skemp, S. Blue Zones: Lessons From the World's Longest Lived. Am. J. Lifestyle 
Med. 2016, 10, 318-321.

40. Veronese, N.; Li, Y.; Manson, J.E.; Willett, W.C.; Fontana, L.; Hu, F.B. Combined associations of body weight and lifestyle factors with all cause and cause specific mortality in men and women: prospective cohort study. BMJ 2016, 355, i5855.

41. Johns, D.J.; Hartmann-Boyce, J.; Jebb, S.A.; Aveyard, P. Diet or Exercise Interventions vs Combined Behavioral Weight Management Programs: A Systematic Review and MetaAnalysis of Direct Comparisons. J. Acad. Nutr. Diet. 2014, 114, 1557-1568.

42. Shaw, K.A.; Gennat, H.C.; O’Rourke, P.; Del Mar, C. Exercise for overweight or obesity. Cochrane Database Syst. Rev. 2006, CD003817.

43. Dwyer-Lindgren, L.; Freedman, G.; Engell, R.E.; Fleming, T.D.; Lim, S.S.; Murray, C.J.; Mokdad, A.H. Prevalence of physical activity and obesity in US counties, 2001-2011: a road map for action. Popul. Health Metr. 2013, 11, 7.

44. Jakše, B.; Pinter, S.; Jakše, B.; Bučar Pajek, M.; Pajek, J. Effects of an Ad Libitum Consumed Low-Fat Plant-Based Diet Supplemented with Plant-Based Meal Replacements on Body Composition Indices. Biomed Res. Int. 2017, 2017, 1-8.

45. Hall, K.D.; Ayuketah, A.; Brychta, R.; Cai, H.; Cassimatis, T.; Chen, K.Y.; Chung, S.T.; Costa, E.; Courville, A.; Darcey, V.; et al. Ultra-Processed Diets Cause Excess Calorie Intake and Weight Gain: An Inpatient Randomized Controlled Trial of Ad Libitum Food Intake. Cell Metab. 2019, 30, 67-77.e3.

46. Campbell, T.C.; Campbell, T.M. The China study: the most comprehensive study of nutrition ever conducted and the startling implications for diet, weight loss and long-term health; BenBella Books, 2005; ISBN 1932100660.

47. Ashley, J.M.; Herzog, H.; Clodfelter, S.; Bovee, V.; Schrage, J.; Pritsos, C. Nutrient adequacy during weight loss interventions: a randomized study in women comparing the dietary intake in a meal replacement group with a traditional food group. Nutr. J. 2007, 6, 12.

48. Johansson, K.; Neovius, M.; Hemmingsson, E. Effects of anti-obesity drugs, diet, and exercise on weight-loss maintenance after a very-low-calorie diet or low-calorie diet: a systematic review and meta-analysis of randomized controlled trials. Am. J. Clin. Nutr. 2014, 99, 14-23.

49. EFSA Scientific Opinion on the substantiation of health claims related to meal replacements for weight control (as defined in Directive 96/8/EC on energy restricted diets for weight loss) and reduction in body weight (ID 1417), and maintenance of body weight a. EFSA J. 2010, 8.

50. Raynor, H.A.; Champagne, C.M. Position of the Academy of Nutrition and Dietetics: Interventions for the Treatment of Overweight and Obesity in Adults. J. Acad. Nutr. Diet. 2016, $116,129-47$.

51. Heymsfield, S.B.; van Mierlo, C.A.J.; van der Knaap, H.C.M.; Heo, M.; Frier, H.I. Weight management using a meal replacement strategy: meta and pooling analysis from six studies. Int. J. Obes. 2003, 27, 537-549.

52. Ross, L.J.; Wallin, S.; Osland, E.J.; Memon, M.A. Commercial Very Low Energy Meal Replacements for Preoperative Weight Loss in Obese Patients: a Systematic Review. Obes. Surg. 2016, 26, 1343-51.

53. Shao, A. Role of Meal Replacements on Weight Management, Health and Nutrition. In Superfood and Functional Food - An Overview of Their Processing and Utilization; InTech, 2017. 
54. NIJZ \& Partners Priloga Specifikacije zahtev za izvedbo ankete v raziskavi »EU Menu Slovenija«»EU Menu Slovenija« POTEK ANKETE MLADOSTNIK / ODRASLI LOT 2; 2019;

55. Hagströmer, M.; Oja, P.; Sjöström, M. The International Physical Activity Questionnaire (IPAQ): a study of concurrent and construct validity. Public Health Nutr. 2006, 9, 755-62.

56. Forde, C. IPAQ scoring protocol - International Physical Activity Questionnaire Available online: https://sites.google.com/site/theipaq/scoring-protocol (accessed on Aug 11, 2019).

57. Ainsworth, B.E.; Haskell, W.L.; Leon, A.S.; Jacobs, D.R.; Montoye, H.J.; Sallis, J.F.; Paffenbarger, R.S. Compendium of physical activities: Classification of energy costs of human physical activities. Med. Sci. Sports Exerc. 1993, 25, 71-74.

58. Lingesh, G.; Khoo, S.; Mohamed, M.N.A.; Taib, N.A.; Group, M. International journal of applied exercise physiology.; 2016; Vol. 5;.

59. Buysse, D.J.; Reynolds, C.F.; Monk, T.H.; Berman, S.R.; Kupfer, D.J. The Pittsburgh Sleep Quality Index: a new instrument for psychiatric practice and research. Psychiatry Res. 1989, 28, 193-213.

60. Levenstein, S.; Prantera, C.; Varvo, V.; Scribano, M.L.; Berto, E.; Luzi, C.; Andreoli, A. Development of the Perceived Stress Questionnaire: a new tool for psychosomatic research. J. Psychosom. Res. 1993, 37, 19-32.

61. Kocalevent, R.-D.; Levenstein, S.; Fliege, H.; Schmid, G.; Hinz, A.; Brähler, E.; Klapp, B.F. Contribution to the construct validity of the Perceived Stress Questionnaire from a populationbased survey. J. Psychosom. Res. 2007, 63, 71-81.

62. Fliege, H.; Rose, M.; Arck, P.; Walter, O.B.; Kocalevent, R.-D.; Weber, C.; Klapp, B.F. The Perceived Stress Questionnaire (PSQ) Reconsidered: Validation and Reference Values From Different Clinical and Healthy Adult Samples. Psychosom. Med. 2005, 67, 78-88.

63. Shahid, A.; Wilkinson, K.; Marcu, S.; Shapiro, C.M. Perceived Stress Questionnaire (PSQ). In STOP, THAT and One Hundred Other Sleep Scales; Springer New York: New York, NY, 2011; pp. 273-274.

64. Bergdahl, J.; Bergdahl, M. Perceived stress in adults: prevalence and association of depression, anxiety and medication in a Swedish population. Stress Heal. 2002, 18, 235-241.

65. Verney, J.; Schwartz, C.; Amiche, S.; Pereira, B.; Thivel, D. Comparisons of a Multi-Frequency Bioelectrical Impedance Analysis to the Dual-Energy X-Ray Absorptiometry Scan in Healthy Young Adults Depending on their Physical Activity Level. J. Hum. Kinet. 2015, 47, 73-80.

66. Wickham, H.; François, R.; Henry, L.; Müller, K. A Grammar of Data Manipulation. R package version 0.8.1. Available online: https://cran.r-project.org/web/packages/dplyr/index.html (accessed on Sep 30, 2019).

67. Wickham, H. Ggplot2: Elegant graphics for data analysis; 2nd ed.; 2016; ISBN 9783319242774.

68. Heinzen, E.; Sinnwell, J.; Atkinson, E.; Gunderson, T.; Votruba, P.; Dougherty, G.; Lennon, R.; Hanson, A.; Goergen, K.; Lundt, E.; et al. An Arsenal of "R" Functions for Large-Scale Statistical Summaries [R package arsenal version 3.3.0] Available online: https://cran.rproject.org/web/packages/arsenal/index.html (accessed on Sep 30, 2019).

69. WHO Body mass index - BMI Available online: http://www.euro.who.int/en/healthtopics/disease-prevention/nutrition/a-healthy-lifestyle/body-mass-index-bmi (accessed on Sep 30, 2019). 
70. WHO Physical Status: The Use and Intepretation of Anthropometry. Report of a WHO Expert Committee; Geneva, 1995;

71. Dyett, P.A.; Sabaté, J.; Haddad, E.; Rajaram, S.; Shavlik, D. Vegan lifestyle behaviors. An exploration of congruence with health-related beliefs and assessed health indices. Appetite 2013, 67, 119-124.

72. Radnitz, C.; Beezhold, B.; DiMatteo, J. Investigation of lifestyle choices of individuals following a vegan diet for health and ethical reasons. Appetite 2015, 90, 31-36.

73. Janssen, M.; Busch, C.; Rödiger, M.; Hamm, U. Motives of consumers following a vegan diet and their attitudes towards animal agriculture. Appetite 2016, 105, 643-651.

74. Pribis, P.; Pencak, R.C.; Grajales, T. Beliefs and attitudes toward vegetarian lifestyle across generations. Nutrients 2010, 2, 523-31.

75. Hoffman, S.R.; Stallings, S.F.; Bessinger, R.C.; Brooks, G.T. Differences between health and ethical vegetarians. Strength of conviction, nutrition knowledge, dietary restriction, and duration of adherence. Appetite 2013, 65, 139-144.

76. Timko, C.A.; Hormes, J.M.; Chubski, J. Will the real vegetarian please stand up? An investigation of dietary restraint and eating disorder symptoms in vegetarians versus nonvegetarians. Appetite 2012, 58, 982-990.

77. Barthels, F.; Meyer, F.; Pietrowsky, R. Orthorexic and restrained eating behaviour in vegans, vegetarians, and individuals on a diet. Eat. Weight Disord. - Stud. Anorexia, Bulim. Obes. 2018, 23, 159-166.

78. Çiçekoğlu, P.; Tunçay, G.Y. A Comparison of Eating Attitudes Between Vegans/Vegetarians and Nonvegans/Nonvegetarians in Terms of Orthorexia Nervosa. Arch. Psychiatr. Nurs. 2018, 32, 200-205.

79. Heiss, S.; Coffino, J.A.; Hormes, J.M. Eating and health behaviors in vegans compared to omnivores: Dispelling common myths. Appetite 2017, 118, 129-135.

80. Barnard, N.D.; Levin, S. Vegetarian Diets and Disordered Eating. J. Am. Diet. Assoc. 2009, 109, 1523.

81. Brandhorst, S.; Longo, V.D. Dietary Restrictions and Nutrition in the Prevention and Treatment of Cardiovascular Disease. Circ. Res. 2019, 124, 952-965.

82. Ornish, D.; Scherwitz, L.W.; Billings, J.H.; Brown, S.E.; Gould, K.L.; Merritt, T.A.; Sparler, S.; Armstrong, W.T.; Ports, T.A.; Kirkeeide, R.L.; et al. Intensive lifestyle changes for reversal of coronary heart disease. JAMA 1998, 280, 2001-7.

83. Ornish, D.; Brown, S.E.; Scherwitz, L.W.; Billings, J.H.; Armstrong, W.T.; Ports, T.A.; McLanahan, S.M.; Kirkeeide, R.L.; Brand, R.J.; Gould, K.L. Can lifestyle changes reverse coronary heart disease? The Lifestyle Heart Trial. Lancet (London, England) 1990, 336, 129-33.

84. Ornish, D.; Weidner, G.; Fair, W.R.; Marlin, R.; Pettengill, E.B.; Raisin, C.J.; Dunn-Emke, S.; Crutchfield, L.; Jacobs, F.N.; Barnard, R.J.; et al. Intensive lifestyle changes may affect the progression of prostate cancer. J. Urol. 2005, 174, 1065-1070.

85. Esselstyn, C.B. Updating a 12-year experience with arrest and reversal therapy for coronary heart disease (an overdue requiem for palliative cardiology). Am. J. Cardiol. 1999, 84, 339-41, A8.

86. Esselstyn, C.B.; Gendy, G.; Doyle, J.; Golubic, M.; Roizen, M.F. A way to reverse CAD? J. Fam. 
Pract. 2014, 63, 356-364b.

87. Rezende, L.F.M.; Sá, T.H.; Mielke, G.I.; Viscondi, J.Y.K.; Rey-López, J.P.; Garcia, L.M.T. AllCause Mortality Attributable to Sitting Time. Am. J. Prev. Med. 2016, 51, 253-263.

88. GBD 2015 Obesity Collaborators; Afshin, A.; Forouzanfar, M.H.; Reitsma, M.B.; Sur, P.; Estep, K.; Lee, A.; Marczak, L.; Mokdad, A.H.; Moradi-Lakeh, M.; et al. Health Effects of Overweight and Obesity in 195 Countries over 25 Years. N. Engl. J. Med. 2017, 377, 13-27.

89. Biddle, S.J.H.; Bennie, J.A.; Bauman, A.E.; Chau, J.Y.; Dunstan, D.; Owen, N.; Stamatakis, E.; van Uffelen, J.G.Z. Too much sitting and all-cause mortality: is there a causal link? BMC Public Health 2016, 16, 635.

90. van Uffelen, J.G.Z.; Wong, J.; Chau, J.Y.; van der Ploeg, H.P.; Riphagen, I.; Gilson, N.D.; Burton, N.W.; Healy, G.N.; Thorp, A.A.; Clark, B.K.; et al. Occupational Sitting and Health Risks. Am. J. Prev. Med. 2010, 39, 379-388.

91. Patel, A. V; Bernstein, L.; Deka, A.; Feigelson, H.S.; Campbell, P.T.; Gapstur, S.M.; Colditz, G.A.; Thun, M.J. Leisure time spent sitting in relation to total mortality in a prospective cohort of US adults. Am. J. Epidemiol. 2010, 172, 419-29.

92. Westerterp, K.R. Exercise, energy balance and body composition. Eur. J. Clin. Nutr. 2018, 72, 1246-1250.

93. Ross, R.; Janssen, I. Physical activity, total and regional obesity: dose-response considerations. Med. Sci. Sports Exerc. 2001, 33, S521-7; discussion S528-9.

94. Thomas, D.M.; Bouchard, C.; Church, T.; Slentz, C.; Kraus, W.E.; Redman, L.M.; Martin, C.K.; Silva, A.M.; Vossen, M.; Westerterp, K.; et al. Why do individuals not lose more weight from an exercise intervention at a defined dose? An energy balance analysis. Obes. Rev. 2012, 13, 83547.

95. Craig, C.L.; Marshall, A.L.; Sjöström, M.; Bauman, A.E.; Booth, M.L.; Ainsworth, B.E.; Pratt, M.; Ekelund, U.; Yngve, A.; Sallis, J.F.; et al. International physical activity questionnaire: 12Country reliability and validity. Med. Sci. Sports Exerc. 2003, 35, 1381-1395.

96. Bauman, A.; Bull, F.; Chey, T.; Craig, C.L.; Ainsworth, B.E.; Sallis, J.F.; Bowles, H.R.; Hagstromer, M.; Sjostrom, M.; Pratt, M.; et al. The International Prevalence Study on Physical Activity: results from 20 countries. Int. J. Behav. Nutr. Phys. Act. 2009, 6, 21.

97. Cleland, C.; Ferguson, S.; Ellis, G.; Hunter, R.F. Validity of the International Physical Activity Questionnaire (IPAQ) for assessing moderate-to-vigorous physical activity and sedentary behaviour of older adults in the United Kingdom. BMC Med. Res. Methodol. 2018, 18, 176.

98. Kyu, H.H.; Bachman, V.F.; Alexander, L.T.; Mumford, J.E.; Afshin, A.; Estep, K.; Veerman, J.L.; Delwiche, K.; Iannarone, M.L.; Moyer, M.L.; et al. Physical activity and risk of breast cancer, colon cancer, diabetes, ischemic heart disease, and ischemic stroke events: systematic review and dose-response meta-analysis for the Global Burden of Disease Study 2013. BMJ 2016, i3857.

99. St-Onge, M.-P.; Mikic, A.; Pietrolungo, C.E. Effects of Diet on Sleep Quality. Adv. Nutr. 2016, 7, 938-49.

100. Medic, G.; Wille, M.; Hemels, M.E. Short- and long-term health consequences of sleep disruption. Nat. Sci. Sleep 2017, 9, 151-161.

101. Beezhold, B.L.; Johnston, C.S. Restriction of meat, fish, and poultry in omnivores improves mood: a pilot randomized controlled trial. Nutr. J. 2012, 11, 9. 
102. Beezhold, B.L.; Johnston, C.S.; Daigle, D.R. Vegetarian diets are associated with healthy mood states: a cross-sectional study in seventh day adventist adults. Nutr. J. 2010, 9, 26.

103. Frank, S.; Gonzalez, K.; Lee-Ang, L.; Young, M.C.; Tamez, M.; Mattei, J. Diet and Sleep Physiology: Public Health and Clinical Implications. Front. Neurol. 2017, 8, 393.

104. St-Onge, M.-P.; Crawford, A.; Aggarwal, B. Plant-based diets: Reducing cardiovascular risk by improving sleep quality? Curr. sleep Med. reports 2018, 4, 74-78.

105. Stranges, S.; Tigbe, W.; Gómez-Olivé, F.X.; Thorogood, M.; Kandala, N.-B. Sleep Problems: An Emerging Global Epidemic? Findings From the INDEPTH WHO-SAGE Study Among More Than 40,000 Older Adults From 8 Countries Across Africa and Asia. Sleep 2012, 35, 1173-1181.

106. Mai, Q.D.; Hill, T.D.; Vila-Henninger, L.; Grandner, M.A. Employment insecurity and sleep disturbance: Evidence from 31 European countries. J. Sleep Res. 2019, 28, e12763.

107. Schüpbach, R.; Wegmüller, R.; Berguerand, C.; Bui, M.; Herter-Aeberli, I. Micronutrient status and intake in omnivores, vegetarians and vegans in Switzerland. Eur. J. Nutr. 2017, 56, $283-293$.

108. Ghesmaty Sangachin, M.; Cavuoto, L.A.; Wang, Y. Use of various obesity measurement and classification methods in occupational safety and health research: a systematic review of the literature. BMC Obes. 2018, 5, 28.

109. Li, Z.; Heber, D. Sarcopenic obesity in the elderly and strategies for weight management. Nutr. Rev. 2012, 70, 57-64.

110. Lemos, T.; Gallagher, D. Current body composition measurement techniques. Curr. Opin. Endocrinol. Diabetes. Obes. 2017, 24, 310-314.

111. Chrysant, S.G.; Chrysant, G.S. The single use of body mass index for the obesity paradox is misleading and should be used in conjunction with other obesity indices. Postgrad. Med. 2019, 131, 96-102.

112. Nuttall, F.Q. Body Mass Index. Nutr. Today 2015, 50, 117-128.

113. Okorodudu, D.O.; Jumean, M.F.; Montori, V.M.; Romero-Corral, A.; Somers, V.K.; Erwin, P.J.; Lopez-Jimenez, F. Diagnostic performance of body mass index to identify obesity as defined by body adiposity: a systematic review and meta-analysis. Int. J. Obes. 2010, 34, 791-799.

114. Spencer, E.A.; Appleby, P.N.; Davey, G.K.; Key, T.J. Diet and body mass index in 38000 EPICOxford meat-eaters, fish-eaters, vegetarians and vegans. Int. J. Obes. 2003, 27, 728-734.

115. Tong, T.Y.; Key, T.J.; Sobiecki, J.G.; Bradbury, K.E. Anthropometric and physiologic characteristics in white and British Indian vegetarians and nonvegetarians in the UK Biobank. Am. J. Clin. Nutr. 2018, 107, 909-920.

116. Fontana, L.; Meyer, T.E.; Klein, S.; Holloszy, J.O. Long-Term Low-Calorie Low-Protein Vegan Diet and Endurance Exercise are Associated with Low Cardiometabolic Risk. Rejuvenation Res. 2007, 10, 225-234.

117. Kristensen, N.B.; Madsen, M.L.; Hansen, T.H.; Allin, K.H.; Hoppe, C.; Fagt, S.; Lausten, M.S.; Gøbel, R.J.; Vestergaard, H.; Hansen, T.; et al. Intake of macro- and micronutrients in Danish vegans. Nutr. J. 2015, 14, 115.

118. Losasso, C.; Eckert, E.M.; Mastrorilli, E.; Villiger, J.; Mancin, M.; Patuzzi, I.; Di Cesare, A.; Cibin, V.; Barrucci, F.; Pernthaler, J.; et al. Assessing the Influence of Vegan, Vegetarian and Omnivore Oriented Westernized Dietary Styles on Human Gut Microbiota: A Cross Sectional Study. Front. Microbiol. 2018, 9, 317. 
119. Welch, A.A.; Shakya-Shrestha, S.; Lentjes, M.A.; Wareham, N.J.; Khaw, K.-T. Dietary intake and status of n-3 polyunsaturated fatty acids in a population of fish-eating and non-fish-eating meat-eaters, vegetarians, and vegans and the precursor-product ratio of $\alpha$-linolenic acid to long-chain n-3 polyunsaturated fatty acids: results from the EPIC-Norfolk cohort. Am. J. Clin. Nutr. 2010, 92, 1040-1051.

120. Benatar, J.R.; Stewart, R.A.H. Cardiometabolic risk factors in vegans; A meta-analysis of observational studies. PLoS One 2018, 13, e0209086.

121. Craig, W.J.; Mangels, A.R. Position of the American Dietetic Association: vegetarian diets. J. Am. Diet. Assoc. 2009, 109, 1266-1282.

122. Keevican, M. California Senate Education Committee Passes Bill Incentivizing Plant-Based Meals in Public Schools Available online: https://www.pcrm.org/news/newsreleases/california-senate-education-committee-passes-bill-incentivizing-plant-based (accessed on Oct 21, 2019).

123. Taylor, J. Brazil's Sustainable School Program Serves Plant-Based Lunches - NYC Food Policy CenterNYC Food Policy Center Available online: https://www.nycfoodpolicy.org/15799-2 (accessed on Oct 20, 2019).

124. St. Germain, C. Vegan Menu Option Requirement, Portugal: Urban Food Policy Snapshot NYC Food Policy CenterNYC Food Policy Center Available online: https://www.nycfoodpolicy.org/vegan-menu-option-requirement-portugal-urban-food-policysnapshot/ (accessed on Oct 20, 2019).

125. Bradbury, K.E.; Guo, W.; Cairns, B.J.; Armstrong, M.E.G.; Key, T.J. Association between physical activity and body fat percentage, with adjustment for BMI: a large cross-sectional analysis of UK Biobank. BMJ Open 2017, 7, e011843.

126. Hollis, J.F.; Gullion, C.M.; Stevens, V.J.; Brantley, P.J.; Appel, L.J.; Ard, J.D.; Champagne, C.M.; Dalcin, A.; Erlinger, T.P.; Funk, K.; et al. Weight loss during the intensive intervention phase of the weight-loss maintenance trial. Am. J. Prev. Med. 2008, 35, 118-26. 